\title{
Coupling Solvent Extraction Units to Cyclic Adsorption Units
}

\author{
Mariana Busto $\mathbb{D}^{1},{ }^{1}$ Enrique Eduardo Tarifa $\mathbb{D}^{\mathrm{D}},{ }^{2}$ and Carlos Román Vera $\mathbb{D}^{1}$ \\ ${ }^{1}$ Institute of Research on Catalysis and Petrochemistry, INCAPE, FIQ-UNL, CONICET, Collecting Ring, National Road 168 Km 0 , \\ El Pozo, 3000 Santa Fe, Argentina \\ ${ }^{2}$ Faculty of Chemical Engineering, Universidad Nacional de Jujuy, CONICET, Ítalo Palanca No. 10, San Salvador de Jujuy, Argentina
}

Correspondence should be addressed to Carlos Román Vera; cvera@fiq.unl.edu.ar

Received 8 November 2017; Revised 19 January 2018; Accepted 24 January 2018; Published 11 March 2018

Academic Editor: Jose C. Merchuk

Copyright (C) 2018 Mariana Busto et al. This is an open access article distributed under the Creative Commons Attribution License, which permits unrestricted use, distribution, and reproduction in any medium, provided the original work is properly cited.

The possibility of regenerating the solvent of extraction units by cyclic adsorption was analyzed. This combination seems convenient when extraction is performed with a high solvent-to-impurity ratio, making other choices of solvent regeneration, typically distillation, unattractive. To our knowledge, the proposed regeneration scheme has not been considered before in the open literature. Basic relations were developed for continuous and discontinuous extraction/adsorption combinations. One example, deacidification of plant oil with alcohol, was studied in detail using separate experiments for measuring process parameters and simulation for predicting performance at different conditions. An activated carbon adsorbent was regenerated by thermal swing, making cyclic operation possible. When extracting the acid with methanol in a spray column, feed $=4 \mathrm{~L} \mathrm{~min}^{-1}, \operatorname{solvent}^{\prime} 80 \mathrm{~L} \mathrm{~min}{ }^{-1}$, feed impurity level $140 \mathrm{mmol} \mathrm{L}^{-1}$, and extract concentration $7.6 \mathrm{mmol} \mathrm{L}^{-1}$, the raffinate reaches a purity of $1.2 \mathrm{mmol} \mathrm{L}^{-1}$, the solvent being regenerated cyclically in the adsorber $(364 \mathrm{~kg})$ to an average of $0.7 \mathrm{mmol} \mathrm{L}^{-1}$. Regeneration of the solvent by cyclic adsorption had a low heat duty. Values of $174 \mathrm{~kJ}$ per litre of solvent compared well with the high values for vaporization of the whole extract phase $\left(1011 \mathrm{~kJ} \mathrm{~L}^{-1}\right)$.

\section{Introduction}

Liquid-liquid extraction (LLE) is an important technique of separation used in many applications of the chemical process industry. Distillation, the workhorse of separation processes, is based on boiling point differences; LLE instead is based on different relative solubilities of solutes in two immiscible, or partially miscible, liquids. Extraction is typically used in cases in which distillation is not cost-effective or directly not possible at all. This is the case when azeotropes are formed, or when volatility differences between components are too low, or when heat-sensitive materials are present that could decompose at the high temperatures of distillation. Also, if the component to be recovered has a very high boiling point or is present in very small concentrations, distillation is not cost-effective.

One of the most important steps in the design of LLE is the choice of the solvent, which must meet several criteria in order to achieve a maximum transfer rate: (i) a high solubility for the solute and low solubility for the feed/raffinate; (ii) a density difference with the carrier higher than $0.15 \mathrm{~g} \mathrm{~cm}^{-3}$; (iii) a medium surface tension (5-30 dyne $\left.\mathrm{cm}^{-1}\right)$; (iv) high resistance to thermal degradation when thermal regeneration is used; (v) a high boiling point and low viscosity, for ease of handling. It is readily apparent that not all criteria can be met and that a careful screening is needed to choose the best solvent from a given set.

One aspect not always conveniently stressed in LLE is that of solvent regeneration. This must be easy and energyefficient. As a consequence, when the solvent is being chosen, it must be decided how is to be regenerated. Since most solvents are regenerated by distillation, aspects to be analyzed are selectivity, solute distribution, and volatility. Solvents that display high selectivity usually have low solute distribution coefficients. If they also have lower volatility than the impurity, the impurity can be recovered as a distillate. However, if the solvent has a lower boiling point than the impurity, then the solvent should be distilled off for regeneration. If the impurity distribution coefficient is low, then a high solvent-to-feed ratio is needed. High solvent recycle rates and 
high solvent regeneration rates are hence needed. For these systems, separation by distillation might not be an option, and other principle could be chosen. An option that has not received attention in the scientific literature or the industrial practice is that of solvent regeneration by adsorption.

The possibilities are analyzed in this work of a process using liquid-liquid extraction for removing impurities from a feed, and adsorption for solvent regenerating the solvent. This combination has not been previously discussed in the open literature. Main features from the kinetic, thermodynamic, and process point of view are considered and discussed; and the main parameters for designing such a process are written. Equations are revised for batch and continuous units involving local and global interphase mass transfer coefficients, and the range of practical values of these parameters for these two operations is discussed.

One example involving experimental work is used as proof-of-concept, deacidification of vegetable oils by extraction with alcohol, coupled to the cyclic adsorption of carboxylic acids from alcoholic solutions in an activated carbon packed column. The liquid-liquid extraction (LLE) of free fatty acids (FFA) from some plant oils has received some attention lately, because fatty acids can be recovered easily for further processing, the yield of neutral oil being maximum [1-3]. These are advantages of LLE over caustic refining in which oil is lost because of reaction with the caustic (saponification) and by emulsification, FFAs being converted to a difficult to handle soap-stock [4]. It has also advantages over physical refining (removal of acids by distillation at $250^{\circ} \mathrm{C}$ ) that produces undesirable changes in color and can also produce the degradation of valuable nutraceuticals and antioxidants [5-7].

Applications of an extraction/adsorption process for refining plant oils could be useful in the biodiesel industry, the food industry, and the industry of biodegradable technical oils (lubricants, dielectric transformer oil, etc.).

\section{Materials and Methods}

The general procedure was as follows: (i) liquid-liquid thermodynamic equilibrium data was got for the solventfeed-impurity system in the form of partition coefficients; (ii) solid-liquid thermodynamic equilibrium isotherms were obtained for the solvent-adsorbent-impurity system; (iii) kinetic parameters for extraction were obtained, in the form of global average $a K_{L}$ values $\left(\mathrm{min}^{-1}\right)$ for a column and stirred tank extractor; (iv) adsorption kinetic parameters for adsorption were obtained, in the form of global average linear driving force parameter $\left(K_{\mathrm{LDF}}\right)$ values $\left(\mathrm{min}^{-1}\right)$; $(\mathrm{v})$ tests of adsorbent regeneration by thermal swing were made, measuring the relevant parameters; (vi) simulations were run for continuous and discontinuous units, varying process conditions.

2.1. Materials. Edible sunflower oil and oleic acid (SigmaAldrich $99 \%$ grade) were used as a source of triglyceride and fatty acid, respectively. Acidified solutions of plant oil of variable concentration were obtained by dissolving weighed amounts of oleic acid in sunflower oil. The solvent used, methanol, was supplied by Biopack (Buenos Aires, Argentina). The chemical purities were higher than 99\%. All compounds were used without further purification. Activated carbon (Filtrasorb, Calgon Carbon) was used in this study. The carbon was conditioned upon receiving by boiling in deionized water for 1 hour, then drying in an oven at $110^{\circ} \mathrm{C}$ for 24 hours. The activated carbon had a BET specific surface area of $972 \mathrm{~m}^{2} \mathrm{~g}^{-1}$, a total pore volume of $0.68 \mathrm{~mL} \mathrm{~g}^{-1}$, and a bulk density of $0.502 \mathrm{~g} \mathrm{~mL}^{-1}$.

2.2. Liquid Extraction Equilibrium. The feed-impurity-solvent system was sunflower oil-oleic acid-methanol. The oleic acid was distributed between the sunflower oil (oil phase) and methanol (alcohol phase). The alcohol and oil phases were mostly immiscible. Experimental LLE data were obtained in a stirred tank reactor. This had an AISI 304 stainless steel vessel with $100 \mathrm{ml}$ total volume, $40 \mathrm{~mm}$ of diameter, and $80 \mathrm{~mm}$ of length and a magnetic coupling between the motor and the stirrer. The tank was heated with a tubular furnace and the temperature was controlled with a Novus N1100 controller. The amounts of each component for preparing the solutions were determined by weighing on an analytical balance (Model Shimadzu AUW220D Dual Range Balance, $0.0001 \mathrm{~g}$ precision). The mixtures were vigorously stirred for $4 \mathrm{~h}$ and then left to rest for at least $12 \mathrm{~h}$. This led to the formation of two clear and transparent phases, with a welldefined interface that were sampled for analysis.

The oleic acid concentration was determined by potentiometric titration (AOCS Method Ca 5a-40) with a microburet. The amount of methanol in the oil phase was determined by weighing the liquid before and after evaporating the solution $\left(80^{\circ} \mathrm{C}, 300 \mathrm{mmHg}\right.$ vacuum). The amount of oil in the methanol phase was determined from a mass balance of the previous components. The analysis was repeated at least three times, and the average of these readings was taken as the liquid phase composition.

2.3. Extraction Kinetics. Values of the average mass transfer coefficient on the solvent side $a K_{\mathrm{MeOH}}$ were calculated from extraction tests in two kinds of extractors: a spray column and a laboratory stirred tank reactor. Coefficients for the column were obtained from single drop experiments using the methodology of Azizi et al. [24].

In the stirred tank tests, the technique of Schindler and Treybal [18] was followed. A stirred tank was used that had the same flange and stirrer as the extraction tests. The internal volume, diameter, and length were also the same as in Section 2.2. The only difference was that the tank had two additional connections for continuous operation. The flowrates of solvent and feed were controlled with peristaltic pumps. The oleic acid concentration in the raffinate and extract phases were determined by titration after adequate settling and formation of two distinct separate phases.

2.4. Adsorption Equilibrium. Adsorption isotherms were measured in a continuously stirred tank batch reactor. The method chosen was that of solid addition in which different 

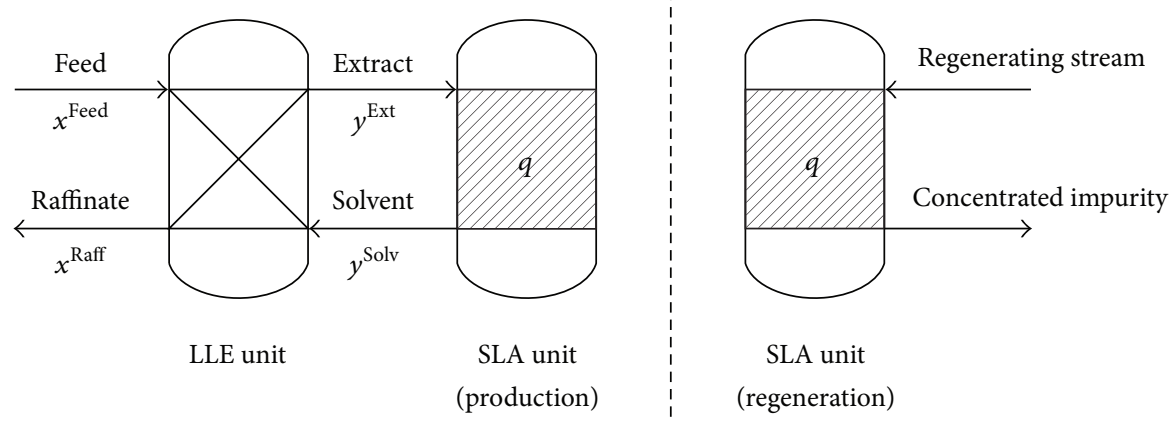

SLA unit

(regeneration)

FIgURE 1: Scheme of liquid-liquid extraction (LLE) unit coupled to solid-liquid adsorption (SLA) unit.

amounts of adsorbent in powder form (about 200 meshes) were allowed to reach equilibrium. The stirring rate was kept at $1600 \mathrm{rpm}$, and the temperature was kept at 30 and $40^{\circ} \mathrm{C}$. The acidity of the methanol solution was determined by potentiometric titration using the average of two measurements. This technique had an average error of $0.69 \%$. Concentration of oleic acid in the solid was determined by a mass balance.

2.5. Adsorption Kinetics. Kinetics of adsorption over the activated carbon in pellet form were measured in a packed bed column with recycle. The mass of the bed was $1 \mathrm{~g}$, and the flowrate of the extract (solvent with dissolved oleic acid) was $7 \mathrm{~L} \mathrm{~h}^{-1}$. The carbon particle size was 35-60 meshes. The mass of the liquid phase was $40 \mathrm{~g}$ and the test lasted $2 \mathrm{~h}$. Samples were taken periodically and oleic acid content of the liquid phase was measured by titration of the acidity as indicated above.

2.6. Settling Tests. Tests of settling rates were made for the sunflower oil-methanol system by vigorously stirring mixtures of varying solvent-to-oil ratio, then being allowed to rest at three different temperatures, 25,40 , and $50^{\circ} \mathrm{C}$. The time was recorded when two distinctive phases were formed and no oil remained in suspension in the upper phase.

\section{Results and Discussion}

3.1. Theoretical Analysis. A scheme of the proposed combination of operations is included in Figure 1. The LLE unit can be a batch or continuous mixer/settler unit, a countercurrent, or cocurrent contact column. The solid-liquid adsorption unit (SLA) can be a bleaching stirred tank or a packed adsorbent column. The latter seems better suited for the proposed combination because it allows an easy separation of the solvent and an easy regeneration of the adsorbent.

The successful matching of the SLA and LLE units seems to relay on the adequate design of the equipment and the choice of solvent and adsorbent. The feed (with impurity concentration $x^{\text {Feed }}$ ) is mixed with the solvent (with impurity concentration $y^{\text {Solv }}$ ) and leaves the contactor, with a lower concentration of impurity, as the raffinate stream

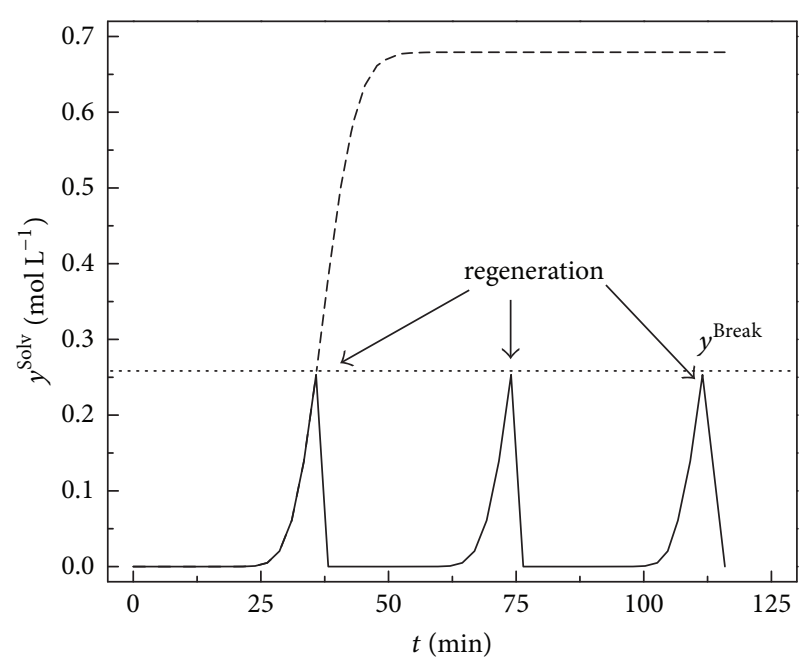

FIGURE 2: SLA unit operated to saturation (dashed line) or in cyclic mode with intermediate regeneration (solid line). Impurity concentration in the fluid phase at the adsorption column outlet, $y^{\text {Solv }}$, as a function of time. Continuous operation. $y^{\text {break }}=$ value of $y^{\text {Solv }}$ at regeneration time.

(of impurity concentration $x^{\text {Raff }}$ ). The extract (with impurity concentration $y^{\mathrm{Ext}}$ ) that leaves the unit must then be fed to the adsorbent column. This column is operated in production mode until the impurity concentration in the exit reaches a limit value ( $\left.y^{\text {break }}\right)$. This is called the breakthrough point. At this point, the feed is stopped, and the column is put into regeneration. The regeneration step can be typically of the thermal type, the column being flushed with a hot fluid to desorb the impurity. For example, a stream of hot solvent can be used, the volume of solvent for regeneration being conveniently small. Figure 2 shows a plot of the concentration of the impurity at the SLA unit exit. The dashed line corresponds to the outlet concentration for the case in which the column is operated to saturation with no intermediate regeneration.

It must be noted that when the column becomes saturated the exit concentration becomes equal to $y^{\mathrm{Ext}}$, the 


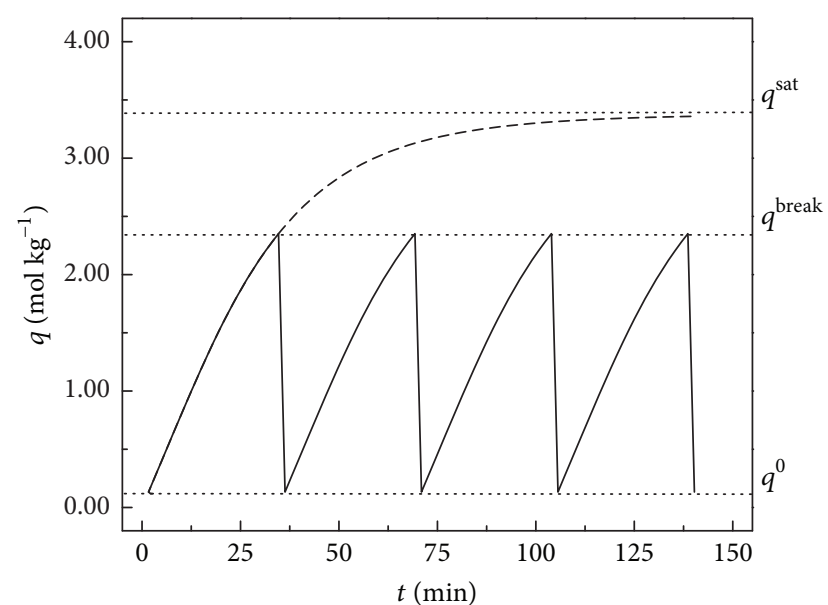

FIGURE 3: Concentration of impurity in the solid phase in an adsorbent column with cyclic regeneration.

concentration at the inlet. The concentration of impurity in the regenerated column is usually not zero, because total regeneration can be rather costly and not practically necessary. As seen in Figure 2, the column also yields a regenerated solvent with variable purity. However, this is not a problem provided $y^{\text {break }}$ is conveniently low.

A similar kind of plot can be seen in Figure 3 but this time the average concentration of the impurity in the solid phase is plotted as a function of time. Again, it can be seen that the column is cycled at a $q^{\text {break }}$ point different to the saturation value. This is because at saturation mass transfer kinetics become too slow due to the decrease in the driving force.

While columns have both a space and time-dependent concentration profiles, other liquid-liquid contactors do not. Stirred tanks with adsorbent in suspension or short packed columns operated with a high liquid recycle have a practically uniform concentration with respect to the spatial coordinate and vary only as a function of time.

The adequate design of the LLE and the SLA units should meet some obvious criteria: (i) the period of operation/regeneration of the column should not be too short, and a minimum value should be specified, for example, 10-40 min; (ii) the adsorbent should have an adequate capacity and adequate adsorption kinetics, with an adequate utilization of the total surface at the point of breakpoint, for example, $40-70 \%$ saturation. The latter is usually a problem for most adsorbents because the internal surface area is very high for materials with small pores, and the mass transfer intrapellet resistance limits the access to the inner pore volume.

A comparison of the mathematical expression for liquidliquid and solid-liquid equilibrium is necessary for understanding the nature of both phenomena. The same can be said for the kinetic expressions for mass transfer between the two phases, either liquid-liquid or liquid-solid. Rather than working with general expressions, the expressions will be written for the practical example: the system of extraction of oleic acid from acidic sunflower oil with methanol and adsorption of oleic acid from methanol over activated carbon. For simplification, the solvent and the feed are supposed to be practically immiscible and that Nernst law is always valid. There is also no reaction involved. For the column equations, plug flow of the individual phases is assumed.

Equation (1) in Table 1 is an example of the isotherm equation for a solid-liquid-adsorbate system in equilibrium and depicts the equilibrium concentration of the impurity on the solid as a function of the concentration in the liquid phase. The function used is that of the Langmuir isotherm. Equation (2) is Freundlich isotherm. Equation (3) is the definition of the partition coefficient for the impurity between the raffinate and extract phases, according to the Nernst law. The coefficient $m$ is a complex function of fluid thermodynamic properties. Nernst law is deduced for low concentrations of solute but can be applied to solutions of higher concentration, though its validity is reduced to a narrower range.

Equations (4)-(6) are the equations for the flux densities (moles per unit area and time) through the liquid-solid interface, while (7)-(9) are the equations corresponding to the transfer to the liquid-liquid interphase. Equations (7)-(9) correspond to the double film model, while (4)-(6) correspond to transfer due to Fickian diffusion on the porous solid side and film diffusion on the liquid side. In (7)-(9), the underlying hypotheses of the double film model apply, that is, the liquid phases are separated by an interface and one film in each phase adheres to this interface. The mass transfer takes place exclusively in this double stagnant film by a molecular diffusion mechanism. In the bulk of each phase the concentration of the impurity is uniform due to perfect mixing.

Equations (10)-(12) and (13)-(16) of Table 1 correspond to flux equations in terms of driving forces and overall mass transfer coefficients. The former are the differences between equilibrium and actual values of concentration at any point in time. In the case of adsorption, the definition of global mass transfer coefficient resembles that of the linear driving force model, $K_{\mathrm{LDF}}$, and hence it will be used as such. The LDF model was first proposed by Glueckauf and Coates [25] as an approximation to mass transfer phenomena in adsorption processes in the gas phase but has been found useful to model adsorption in packed beds because it is simple and consistent both analytically and physically [26]. Several authors have inspected the nature of $K_{\mathrm{LDF}}$. Ruthven and Farooq [27] considered that it is composed of two contributions, related to the intrapellet mass transfer resistance $\left(R_{D}\right)$ and the film mass transfer resistance $\left(R_{f}\right)$, the explicit formulation being that of (17). While $R_{f}$ depends on the diffusivity of the impurity in the fluid phase, $R_{D}$ depends on the diffusivity of the impurity inside the porous matrix of the solid adsorbate. Hence, in most cases, and particularly in adsorption in liquid phase, $R_{D}$ is the highest resistance and $R_{f}$ can be neglected.

Equations (18) and (19) depict the relations between the local liquid-liquid mass transfer coefficients and the overall coefficients. The latter can be expressed in terms of driving forces in the raffinate or extract side leading to two different coefficients. For the local coefficients, depending on which phase is continuous and which is disperse, different 
TABLE 1: Relation between SLA and LLE equilibrium and kinetic coefficients. Application to the extraction of oleic acid with methanol from sunflower oil and the adsorption of oleic acid from methanol over a solid adsorbent (activated carbon).

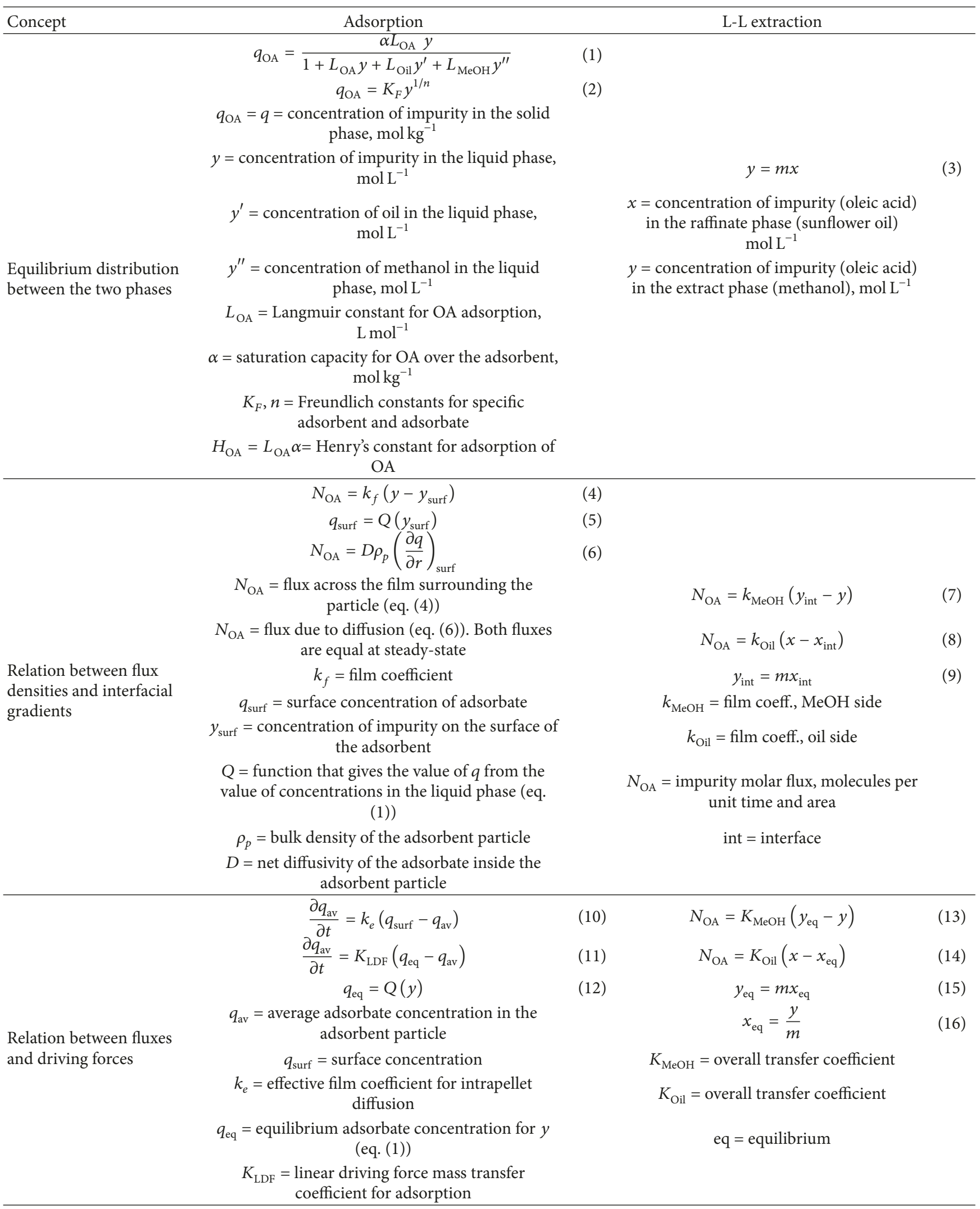


TABle 1: Continued.

\begin{tabular}{|c|c|c|c|c|}
\hline Concept & Adsorption & & L-L extraction & \\
\hline Mass transfer coefficients & $\begin{array}{c}\frac{1}{K_{\mathrm{LDF}}}=R_{f}+R_{D}=\frac{r_{p}}{3 k_{f}}+\frac{r_{p}^{2}}{15 \varepsilon D} \\
\varepsilon=\text { porosity of the adsorbent particle } \\
r_{p}=\text { radius of the adsorbent particle } \\
k_{f}=\text { film transfer coefficient. } \\
R_{f}=\text { film transfer resistance } \\
R_{D}=\text { intrapellet diffusion resistance }\end{array}$ & $(17)$ & $\begin{array}{c}\frac{1}{K_{\mathrm{MeOH}}}=\frac{1}{k_{\mathrm{MeOH}}}+\frac{m}{k_{\mathrm{Oil}}} \\
\frac{1}{K_{\mathrm{Oil}}}=\frac{1}{k_{\mathrm{Oil}}}+\frac{1}{m k_{\mathrm{MeOH}}}=\frac{1}{m K_{\mathrm{MeOH}}} \\
S h_{c}=0.725 S_{c}^{0.42} \Re e_{c}^{0.57}(1-\phi) \\
S h_{c}=\frac{k_{c} d_{32}}{D_{c}} \\
\Re e_{c}=\frac{\rho_{c} d_{32} v_{\text {slip }}}{\mu_{c}} \\
\quad S c_{c}=\frac{\mu_{c}}{D_{c}} \\
k_{d}=0.023 v_{\text {slip }} S c_{c}^{-0.5} \\
\phi=\frac{V_{d}}{V_{d}+V_{c}} \\
\phi=\text { hold-up of the disperse phase (oil) } \\
d_{32}=\text { average Sauter diameter } \\
v_{\text {slip }}=\text { slip velocity between phases } \\
\mu_{c}=\text { viscosity of the continuous phase } \\
V_{d}=\text { volume of the disperse phase } \\
V_{c}=\text { volume of the continuous phase } \\
k_{c}=\text { mass transfer coefficient, continuous } \\
\text { phase } \\
k_{d}=\text { mass transfer coefficient, disperse } \\
\text { phase }\end{array}$ & $\begin{array}{l}(18) \\
(19) \\
(20) \\
(21)\end{array}$ \\
\hline $\begin{array}{l}\text { Mass balance: batch unit } \\
\text { perfectly mixed }\end{array}$ & $\begin{array}{c}V \frac{d y}{d t}=W K_{\mathrm{LDF}}\left(q_{\mathrm{eq}}-q_{\mathrm{av}}\right) \\
y=y^{0}, q=q^{0}, t=0 \\
\frac{d y}{d t}=K_{\mathrm{LDF}}^{\prime}\left(y-y_{\mathrm{eq}}\right) \\
q_{\mathrm{av}}=Q\left(y_{\mathrm{eq}}\right) \\
V=\text { volume of adsorbent } \\
W=\text { weight of adsorbent }\end{array}$ & $\begin{array}{l}(26) \\
(27) \\
(28) \\
(29)\end{array}$ & $\begin{array}{c}\frac{d y}{d t}=\frac{a K_{\mathrm{MeOH}}}{1-\phi}\left(y_{\mathrm{eq}}-y\right) \\
\frac{d x}{d t}=\frac{a K_{\mathrm{Oil}}}{\phi}\left(x-x_{\mathrm{eq}}\right) \\
x=x^{0}, y=y^{0}, t=0 \\
a=\text { interfacial area per unit volume of } \\
\text { whole liquid phase }\end{array}$ & $\begin{array}{l}(30) \\
(31)\end{array}$ \\
\hline $\begin{array}{l}\text { Mass balance: continuous } \\
\text { contact tower equations }\end{array}$ & $\begin{array}{c}\frac{\partial y}{\partial t}-D_{L} \frac{\partial^{2} y}{\partial z^{2}}+\frac{\partial(u y)}{\partial z}+\frac{1-\varepsilon_{B}}{\varepsilon_{B}} \rho_{p} \frac{\partial q}{\partial t}=0 \\
y(0, t)=y^{0} \\
\frac{\partial y}{\partial z}=0, z=L \\
y(z, 0)=0 \\
\varepsilon_{B}=\text { bed porosity } \\
q=\text { fluid phase concentration of the impurity } \\
q=\text { solid phase concentration of the impurity } \\
u=\text { interstitial velocity }=v / \varepsilon_{B} \\
\rho_{p}=\text { particle density } \\
z=\text { axial coordinate } \\
L=\text { height of the column }\end{array}$ & $(34)$ & $\begin{array}{c}z=\int_{x_{1}}^{x_{2}} \frac{v_{\mathrm{oil}} d x}{a_{\mathrm{av}} K_{\mathrm{Oil}}\left(x-x_{\mathrm{eq}}\right)} \\
z=\int_{y_{1}}^{y_{2}} \frac{v_{\mathrm{MeOH}} d y}{a_{\mathrm{av}} K_{\mathrm{MeOH}}\left(y_{\mathrm{eq}}-y\right)} \\
v=\text { superficial velocity } \\
z=\text { axial coordinate, height of the column } \\
a_{\mathrm{av}}=\text { average interfacial area per unit } \\
\text { volume of the contactor vessel }\end{array}$ & $(36)$ \\
\hline
\end{tabular}


correlations will be applied to each side, though both are usually of the form of an equation of the Sherwood number as a function of the Reynolds number, the Schmidt number, and the holdup of the disperse phase. Example equations for calculating $k_{c}$ (local coefficient for the continuous phase) and $k_{d}$ (coefficient for the disperse phase) for column continuous contactors have been written in (20)-(25) according to the suggestions of Koncsag and Barbulescu [28].

Discontinuous operation in perfectly mixed units shows more similarities for both operations as it can be seen in (26) to (31) in Table 1. This is also a problem easily handled mathematically and corresponds to the operation of stirred tank extraction units and stirred tank adsorption units. Packed columns operated with a high recycle ratio can also be considered as perfectly mixed stirred tanks. In (30) and (31), $a$ is the interfacial area per unit equipment volume. Alternatively, the balance in the liquid phase can be written in terms of a $K_{\mathrm{LDF}}^{\prime}$ coefficient, as in (28) and (29).

Equations (32) to (37) correspond to the differential equations, border, and initial conditions that express the mass balance for the adsorbate species during the movement along the packed bed and diffusion inside the porous adsorbent. The full model takes into account the backmixing in the axial direction by considering a Fickian diffusion term with axial diffusivity $D_{L}$. These equations must be coupled to a local equation like (26) that describes the law of variation of $q$ as a function of the process variables.

Equations (36) and (37) are the integrated forms of the local mass balance. They are simpler to handle than the previous one for adsorption. However, this is a simplified view, and more sophisticated models are needed to reflect phenomena of emulsion formation and collapse, carryover, flooding, drop coalescence and breakage, and so forth.

The interfacial area during extraction is a function of the drop size. The drop size is bigger at higher values of holdup of the disperse phase and at bigger values of the stirring power (in stirred tanks). However, the dependence is soft.

The inspection of (26) to (29) and (30) and (31), and their comparison with (32) and (33), shows that adsorption columns can never work in a true steady state like liquidliquid extraction units do. To describe their operation, a solution as a function of time and space must be found. This is because the solid phase is fixed while the fluid phase is flowing continuously. As shown in Figure 2 when the breakthrough condition of the column is reached, the operation of the adsorber must be stopped and regeneration must be performed. This is different from the extraction column in which a continuous steady state can always be established between the two flowing liquid phases.

For both adsorption and extraction, the throughput for any separation unit is mainly given by intrinsic parameters such as the kinetic mass transfer parameters, the thermodynamic constants for the L-L and S-L equilibrium, the parameters describing the interface, and the total volume of the phases. For any given choice of contacting device and set of process conditions, that is, temperature, pressure, and liquid phase flowrates, the volume of the unit would be the result of a design procedure for a given desired rate of extraction and adsorption, because the process conditions will dictate the values of the intrinsic parameters. Therefore, it is of interest to list the range of values of the most important intrinsic parameters involved in the design of adsorption and extractors. This is done in Table 2.

Equivalent coefficients have been placed in the same row. In the case of the interfacial area for adsorption, all available surface area, external and intrapellet, has been included. It must be noted that, due to diffusional resistance, not all surface is readily available. However, this is taken into account when calculating the intrapellet mass transfer resistance. Since, for mesoporous and microporous adsorbents, the inner surface is much higher than external one, $a$ is practically the intrapellet area divided by the pellet volume. It is apparent from this comparison that the S-L interfacial area is much higher than the L-L for most adsorbents and L-L contactors.

Inspection of the last row of Table 2 yields the most important insight. If $K_{\mathrm{LDF}}^{\prime}$ and $a K_{L}$ values are compared, this is a comparison of parameters with similar driving forces, it can be seen that in global terms adsorption is slower than extraction under most conditions, especially for the case of extraction in stirred tanks or static mixers. More similar values are obtained when we match adsorption with a low energy extraction operation, for example, in a spray column. For the coupling of both units however what it must be similar is the uptake of impurity per unit time and this is a function also of the driving force. In this sense, slow adsorption kinetics can be compensated by high solid affinities (high $H, q_{\text {eq }}$ values), while fast extraction kinetics could be inhibited by low impurity solubilities (low $m$ values). All these considerations will have a better insight once the examples are discussed in detail.

3.2. Example of Plant Oil Deacidification. In this example, sunflower oil is first extracted with methanol in order to remove the impurities, that is, oleic acid. The solvent is then regenerated by adsorption of oleic acid from the methanol solution. The adsorbent is in turn regenerated by a thermal swing. In order to obtain sound values of the parameters that describe the phenomena, separate experiments for determining the liquid-liquid and liquid-solid thermodynamic and kinetic parameters were performed.

\subsubsection{Determination of the Partition Coefficient for Oleic Acid.} For the system oleic acid-methanol-sunflower oil, values of $m$ were obtained from plots of $y$ as a function of $x$ at three different temperatures (Figure 4). $m$ was found to be equal to 0.875 at $30^{\circ} \mathrm{C}, 0.922$ at $40^{\circ} \mathrm{C}$, and 1.125 at $50^{\circ} \mathrm{C}$. $m$ was calculated as the ratio of the concentration of oleic acid in the alcohol phase (free of oil) to the concentration of oleic acid in sunflower oil (free of methanol). The concentration of oleic acid in either phase was really a little lower due to dissolution of methanol in the oil phase and dissolution of oil in the alcohol phase. In this sense, the higher solubility of oleic acid in methanol at higher temperatures is also accompanied by a higher solubility of the oil, and hence there must be a balance when choosing the right temperature of operation, because the relative purity of the extract or the yield of raffinate can 


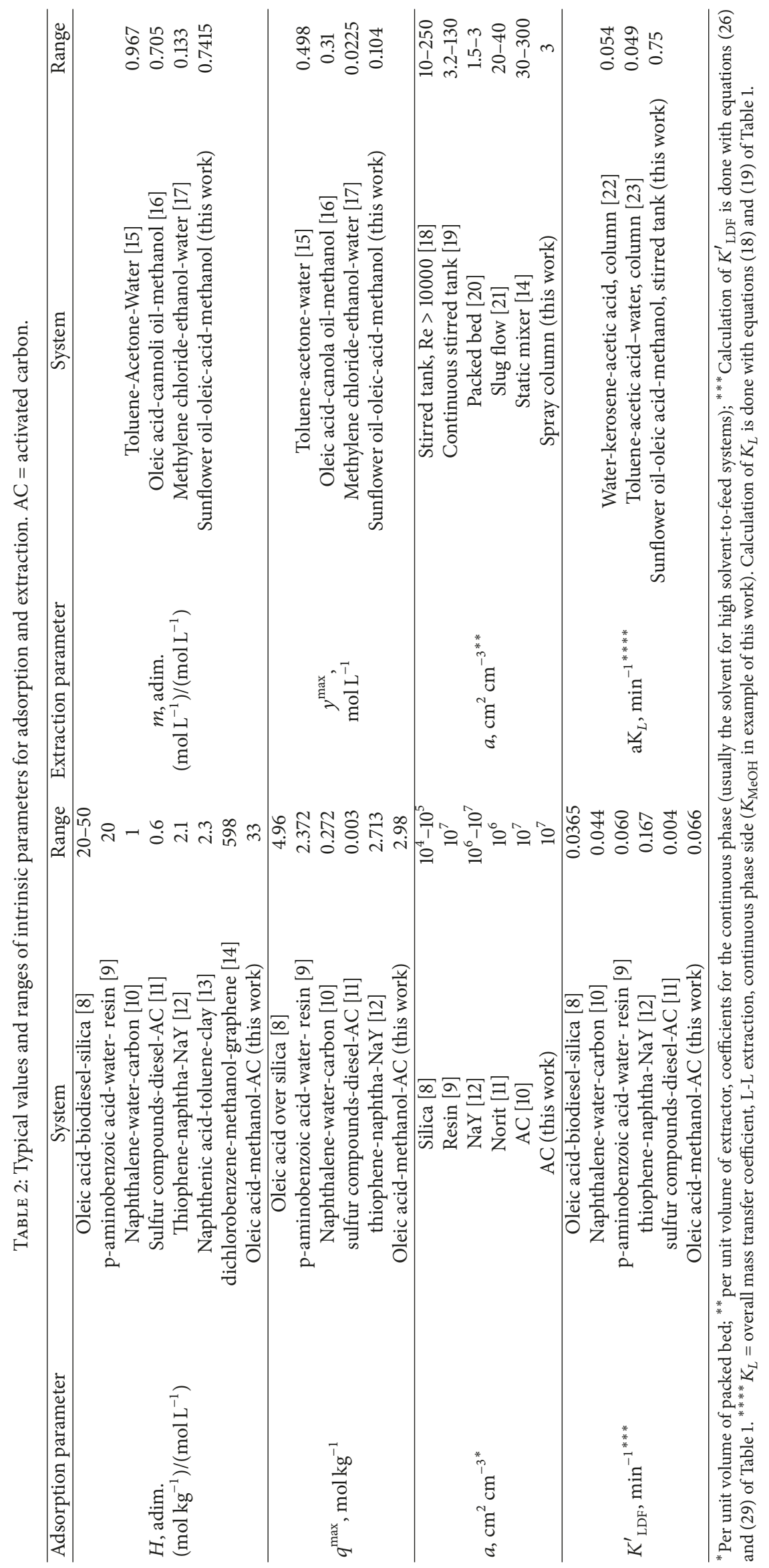


TABLE 3: Experimental values of drop size $(d)$, terminal velocity $(V)$ and mass transfer coefficients (continuous phase side) $\left(a K_{L}\right)$ at three different temperatures. Single drop tests, $\phi=0.15$.

\begin{tabular}{lcccc}
\hline$T,{ }^{\circ} \mathrm{C}$ & $d, \mathrm{~mm}$ & $a, \mathrm{~cm}^{2} \mathrm{~cm}^{-3}$ & $V, \mathrm{~cm} \mathrm{~s}^{-1}$ & $a K_{L}, \mathrm{~min}^{-1}$ \\
\hline 30 & 3.19 & 18.8 & 6.21 & 0.842 \\
40 & 3.13 & 19.2 & 4.93 & 1.190 \\
50 & 3.13 & 19.2 & 3.13 & 1.610 \\
\hline
\end{tabular}

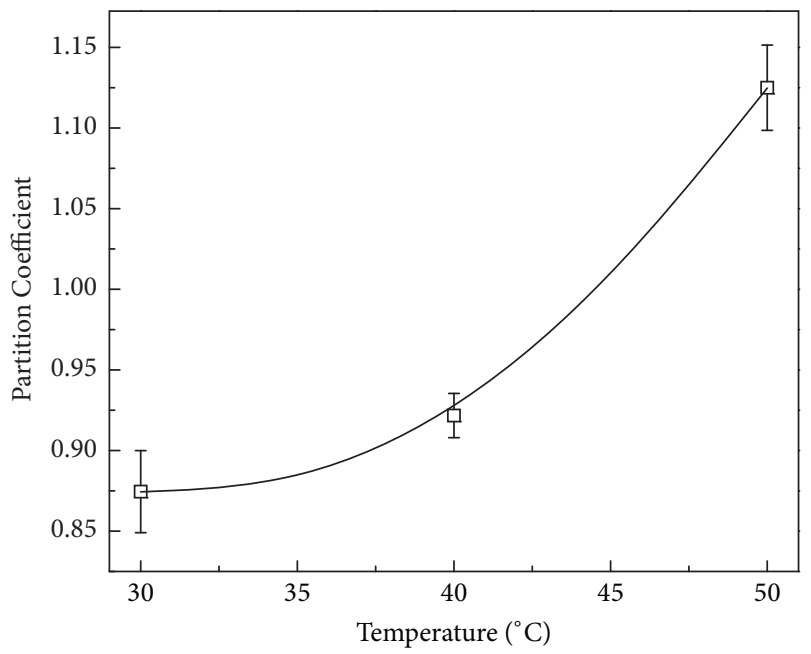

Figure 4: Plot of the partition coefficient as a function of the temperature of the experiment.

be an issue. The linearity of the $y$ versus $x$ plots was very good with $r^{2}$ values of about 0.997 .

\subsubsection{Determination of Mass Transfer Coefficient of Oleic} Acid between Methanol and Sunflower Oil. Mass transfer coefficients varied widely depending on the type of contact equipment used. Results are presented in Table 3 for spray column single drops experiments. Average diameter values were calculated with Sauter's formula (see (38)). Interfacial area is calculated with (39). In the case of the column, increasing temperature values (from 30 to $50^{\circ} \mathrm{C}$ ) increased the overall mass transfer coefficient by almost a factor of 2. This is possibly related to the decrease in viscosity and a significant increase of the Reynolds number (Re).

$$
\begin{gathered}
d_{32}=\sum \frac{n_{i} d_{i}^{3}}{n_{i} d_{i}^{2}}, \\
a=\frac{6 \phi}{d_{32}} .
\end{gathered}
$$

The values obtained compared fairly well with others reported. Sankarshana et al. [29] found values of $a K_{L}$ (continuous phase side, feed/solvent $=0.2-1$ ) equal to $0.02-0.06 \mathrm{~min}^{-1}$ for packed columns with random and ordered packing, while working with a system of acetic acid-ethyl acetate-water system under countercurrent mode. Nosratinia et al. [30] found $a K_{L}$ values of $0.12-0.84 \mathrm{~min}^{-1}$ in a spray column with jet injection of the disperse phase.
Geankoplis and Hixson [31] found values of overall $a K_{L}$ (water, continuous phase side) of $0.07-1.2 \mathrm{~min}^{-1}$ at varying disperse phase flow rates, in a ferric chloride-isopropyl ether$\mathrm{HCl}_{(\mathrm{aq})}$ system, in a spray tower.

In spray towers for liquid-liquid extraction, the Sauter diameter is a function of the disperse phase holdup and fluid dynamic conditions. Salimi-Khorshidi et al. [32] found that $d_{32}$ varied within $2.5-4 \mathrm{~mm}$ when varying $\phi=0.1-0.6$ and flowrates, a volcano plot being found for $d_{32}$ as a function of $\operatorname{Re}$ or $\phi$.

Considerations for the scale-up of $a K_{L}$ coefficients from single drop measurements to full-scale drop swarms should be discussed. Hughmark [33] studied comprehensive data sets, with $\phi=0.006-0.2$, and early found that, for ratios of the continuous to disperse phase viscosity less than one, the mass transfer coefficients (in the form of $\mathrm{Sh}_{c}$ or $k_{c}$ ) for the continuous phase of drop swarms were the same as for single drops, while, for viscosity ratios greater than one, the multiple drop coefficients were somewhat smaller. Hughmark fitted his data with Ruby and Elgin $k_{c}$ equation [34]. In this system, the coefficient $a K_{L}$ is thus a function of the impeller Reynolds and also directly proportional to $\phi$.

The value of the mass transfer coefficient for the stirred tank experiment was $0.75 \mathrm{~min}^{-1}$ using an experimental setup similar to that of Schindler and Treybal [18] and using a holdup of disperse phase of 0.5 . These authors early correlated the mass transfer coefficients for stirred tanks studying the mass transfer between two liquid phases in an agitated baffled vessel and found that mass transfer coefficient increased with impeller Reynolds number and disperse phase holdup. Baffling roughly increased $a K_{L}$ by 1.5 times. Average volumetric $a K_{L}$ values for the continuous phase ranged within $3-25 \mathrm{~min}^{-1}$ for values of the impeller Reynolds number of 20000-60000. The dependence of $a K_{L}$ on impeller Reynolds number was strong, being roughly proportional to $\mathrm{Re}^{2-2.5}$, while the dependence on holdup of the disperse phase was weaker, being proportional to about $\phi^{0.9-1}$. They also found that $k_{c}$ was almost insensitive to variations in the holdup. These trends can be easily rationalized by considering that, for stirred tanks the Sauter diameter, as in (38), is imposed by the impeller Reynolds, while the interfacial area per unit volume of disperse phase corresponds to the value given by (39). For this reason, values of $a K_{L}$ for the simulations will be extrapolated from experimental data at similar stirring conditions by scaling with the value of $\phi$.

3.2.3. Adsorption Properties. For the oleic acid-methanolsunflower oil system, an adsorbent of activated carbon was chosen because of its good performance in preliminary screening tests. This is a fairly novel application for carboxylic acid adsorption since, in the literature, silica, silicates, clays, and zeolites have usually been employed [35-37] while reports on the use of carbons are concentrated on decontamination of water [38, 39].

Results of adsorption of oleic acid in sunflower oil overactivated carbon are plotted in Figure 5. The curves correspond to a virgin activated carbon. The last value in the abscissae axis is $0.10 \mathrm{~mol} \mathrm{~L}^{-1}$; therefore the plotted results 


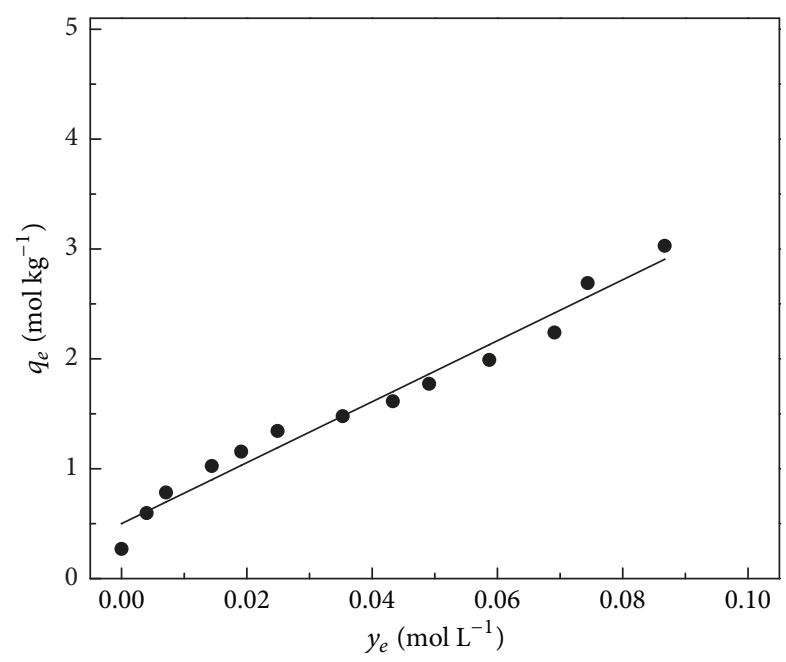

FIGURE 5: Adsorption of oleic acid from methanol at room temperature $\left(30^{\circ} \mathrm{C}\right)$ and overactivated carbon (Calgon Carbon Filtrasorb 200). Correlation coefficient for Henry's constant (slope of the line), $r^{2}=0.97$.

correspond to mildly acidic sunflower oil and sunflower oil of low acid content. The curve is better interpreted by the Freundlich model (Table 1, equation (2)), with $K_{F}=8.34$ and $n=1.66$, with an $r^{2}=0.971$. For simplicity of the treatment however, the data can be fitted with Henry's linear isotherm yielding $H=33\left(\mathrm{~L} \mathrm{~kg}^{-1}\right)$.

Another isotherm was taken at $40^{\circ} \mathrm{C}$ in order to calculate the heat of adsorption. This isotherm had an $H$ value of $16\left(\mathrm{~L} \mathrm{~kg}^{-1}\right)$. Applying the integrated form of van't Hoff equation and considering that the heat of adsorption was not a function of temperature, the heat of adsorption was estimated as $57 \mathrm{~kJ} \mathrm{~mol}^{-1}$. This value compares well with other found in the literature. Li et al. [40] found that adsorption of phenol on resin from aqueous solutions had a heat of adsorption of about $38 \mathrm{~kJ} \mathrm{~mol}^{-1}$. Chiou and $\mathrm{Li}$ [41] found a heat of adsorption of $52.9 \mathrm{~kJ} \mathrm{~mol}^{-1}$ for reactive dye in aqueous solution on chemical cross-linked chitosan beads. Ilgen and Dulger [42] measured a value of about $34 \mathrm{~kJ} \mathrm{~mol}^{-1}$ for the adsorption of oleic acid from sunflower oil over zeolite $13 x$.

Adsorption tests were also made in a packed bed column with fast recycle. In this column the axial concentration gradient was negligible and the behavior was similar to a stirred tank with perfect mixing. The results were fitted with the simple model of the linear driving force model, in the form of (26). The results for one of such tests are plotted in Figure 6 . The calculated value for $K_{\mathrm{LDF}}$ from the experiment is $0.066 \mathrm{~min}^{-1}$.

3.2.4. Settling Times for Phase Separation in a Gravity Decanter. The results of the experiments to measure the settling time as a function of the volumetric methanol-tooil ratio and the temperature are included in Figure 7. At $25^{\circ} \mathrm{C}$ for methanol-to-oil ratios lower than 1 no complete phase separation could be achieved even after 1 day, some oil

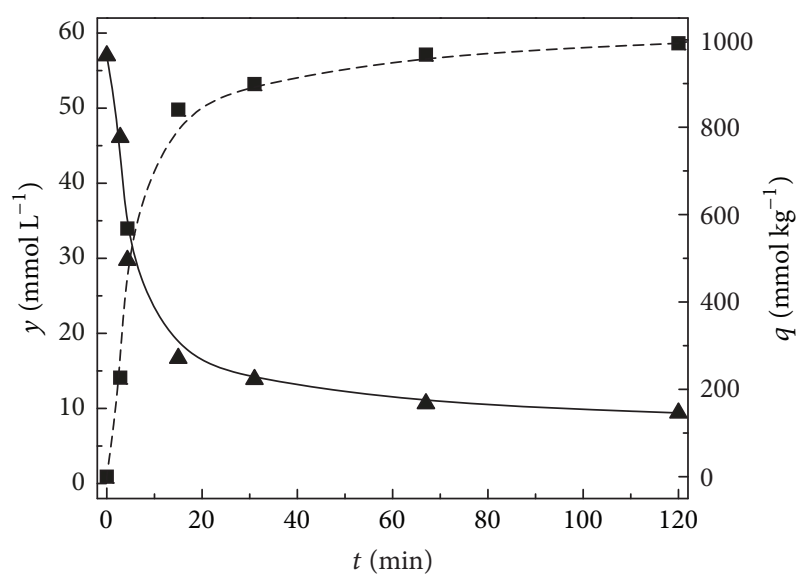

FIGURE 6: Concentration of oleic acid in both the liquid phase $(y, \mathbf{\Delta})$ and solid phase $(q, \boldsymbol{\square})$ as a function of time. Packed column with fast recycle, $30^{\circ} \mathrm{C}$. Granular activated carbon, average pellet diameter, $0.4 \mathrm{~mm}$.

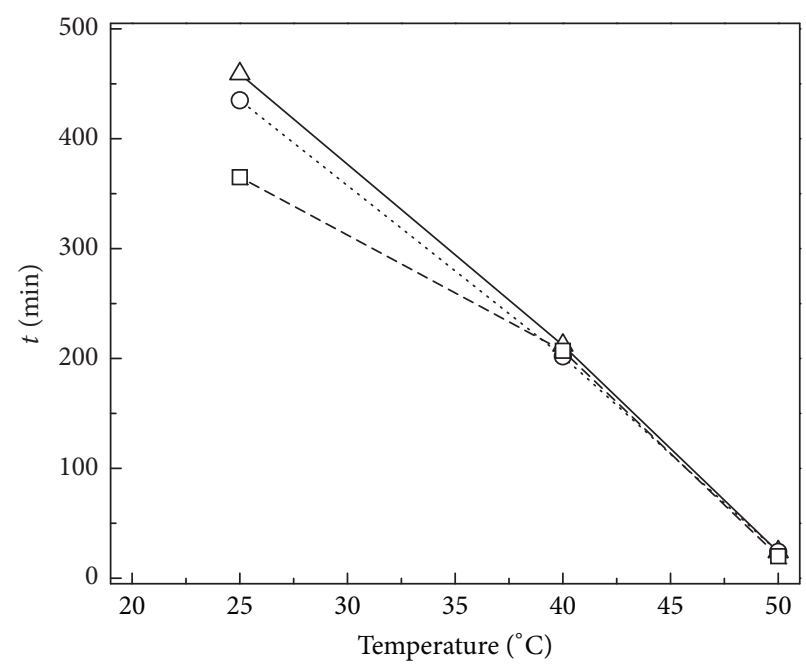

FIGURE 7: Experimental settling time as a function of the temperature. Tank with no internals and no coalescing aid. Disperse phase (oil) holdup: $0.5(\triangle), 0.25(\bigcirc)$, and $0.2(\square)$.

remaining disperse in the methanol phase as indicated by the opacity of the upper phase.

Complete separation was achieved for methanol-to-oil ratios equal to or higher than 1 . The general trends were that, at high temperatures, for example, $50^{\circ} \mathrm{C}$, the settling time was independent of the methanol-to-oil ratio, while at lower temperatures higher methanol-to-oil ratios lowered the settling time. For a continuous operation of a decanter with a settling time of one hour, a temperature of about $50^{\circ} \mathrm{C}$ is needed.

\subsubsection{Simulation}

(1) Simulation of a Continuous Extraction/Adsorption Process. The layout of a process using an extraction column coupled to a set of twin adsorption columns is depicted in Figure 8. 


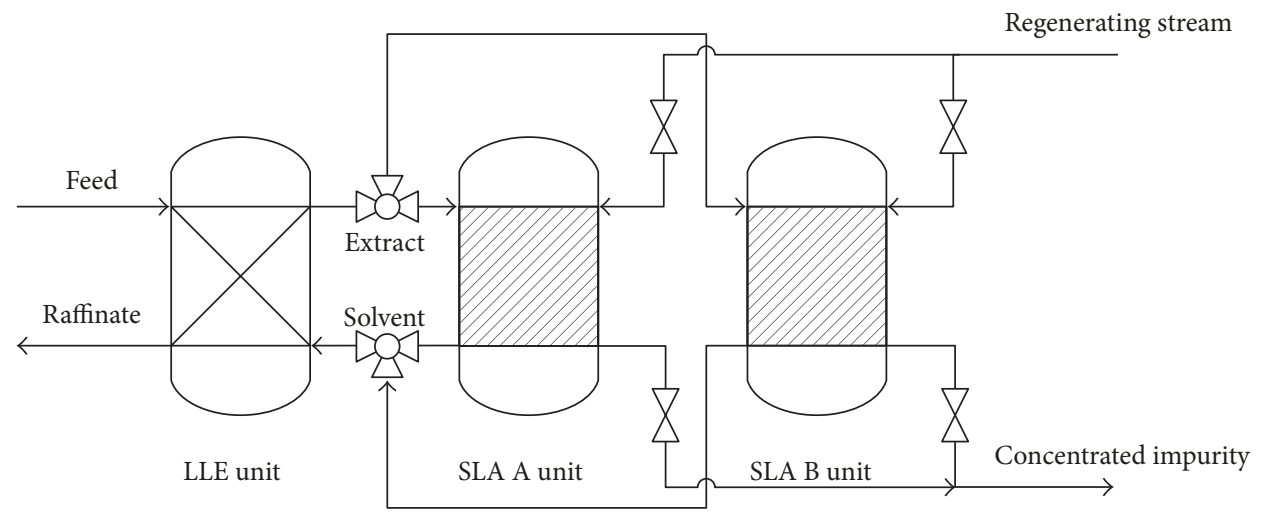

FIGURE 8: Flowsheet of extraction column coupled to a set of twin adsorption columns. $W^{\text {Ads }}=364 \mathrm{~kg}, V^{\text {Ext }}=0.15 \mathrm{~m}^{3}$.

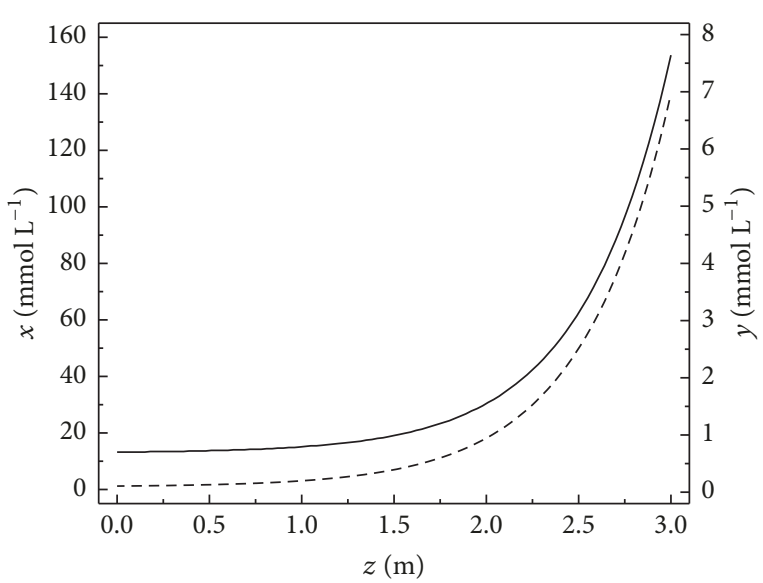

Figure 9: Plot of $x$ (dashed line) and $y$ (solid line) as a function of the position in the countercurrent extraction column. $Q^{\text {Feed }}=$ $4 \mathrm{~L} \mathrm{~min}^{-1}, Q^{\text {Solv }}=80 \mathrm{~L} \mathrm{~min}^{-1}, x^{\text {Feed }}=0.140 \mathrm{~mol} \mathrm{~L}^{-1}, y^{\text {Solv }}=$ $0.7 \mathrm{mmol} \mathrm{L}^{-1}$.

In this layout the operation of the extraction column is continuous, and the equations describing the relation between the concentration in any phase and time are (32)-(35) in the case of the adsorption column. The equations describing the exchange in the case of extraction are (36) and (37). The solution of this system of equations for an example of extraction of acidic sunflower oil with methanol is depicted from Figures 9-11.

The holdup of the disperse phase was calculated from the experimentally measured characteristic velocity of the drop $\left(v_{K}\right)$, and the values of the flowrates of the feed and solvent, by means of the equation of Gayler (40). Gayler proposed that for many different types of columns the following equation held [43]:

$$
\frac{u_{d}}{\phi}+\frac{u_{c}}{1-\phi}=u_{k}(1-\phi)
$$

where $u_{c}$ and $u_{d}$ are the superficial velocities of the continuous and dispersed phases, respectively, and $u_{k}$, the characteristic velocity, is the mean relative velocity of droplets extrapolated to zero flowrate and can be identified with

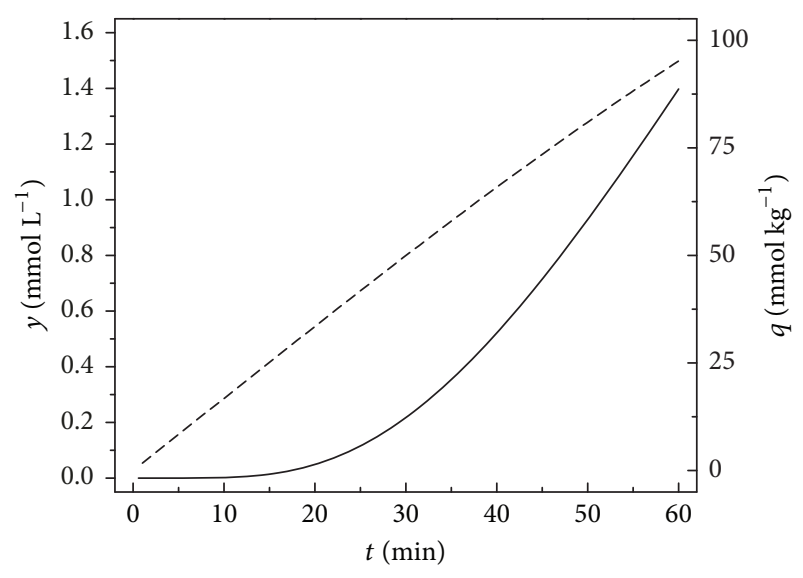

FIGURE 10: Adsorption column. Plot of $y$ (solid line) and $q$ (dashed line) as a function of time. $W^{\mathrm{Ads}}=364 \mathrm{~kg}, Q^{\mathrm{Ext}}=80 \mathrm{~L} \mathrm{~min}^{-1}, y^{\mathrm{Ext}}=$ $7.64 \mathrm{mmol} \mathrm{L}^{-1}, q_{0}=0 \mathrm{~mol} \mathrm{~kg}{ }^{-1}$.

the terminal velocity of a single drop in the equipment concerned. Equation (40) was numerically solved, giving $\phi=$ 0.044 .

The equations of the column extraction unit were solved analytically in order to avoid the problem of solving the twopoint boundary value problem imposed by the countercurrent flow. Equations (36) and (37) were solved by obtaining the eigenvalues and eigenvectors of the matrix of derivatives and by considering that the solution eigenfunction was $y=$ $k \exp (\lambda z)$. One eigenvalue was found to be zero, so both $x$ and $y$ had the form $g=A+B \exp (\lambda z)$, where $\lambda$ is a function of $m$, $v^{\text {Feed }}, v^{\text {Solv }}$, and $a K_{L}$; and $A$ and $B$ are function of the previous parameters and also the initial conditions.

The impurity concentration in the feed, $140 \mathrm{mmol} \mathrm{L}^{-1}$, is equivalent to about $4.4 \%$ acidity, which should be reduced to about $30 \mathrm{mmol} \mathrm{L}^{-1}$ in order to be suitable as a feed for the biodiesel alkali-catalyzed process. For some applications, the maximum acidity is even lower. For insulating oils, ASTM D3487 establishes a maximum acidity of $0.03 \mathrm{mg} \mathrm{KOH} \mathrm{g}^{-1}$, that is, $0.5 \mathrm{mmol} \mathrm{L}^{-1}$. This is near the concentration value of the raffinate in Figure 9, $1.1 \mathrm{mmol} \mathrm{L}^{-1}$. Anyway, a special limit for insulating oils of plant origin is established in ASTM 


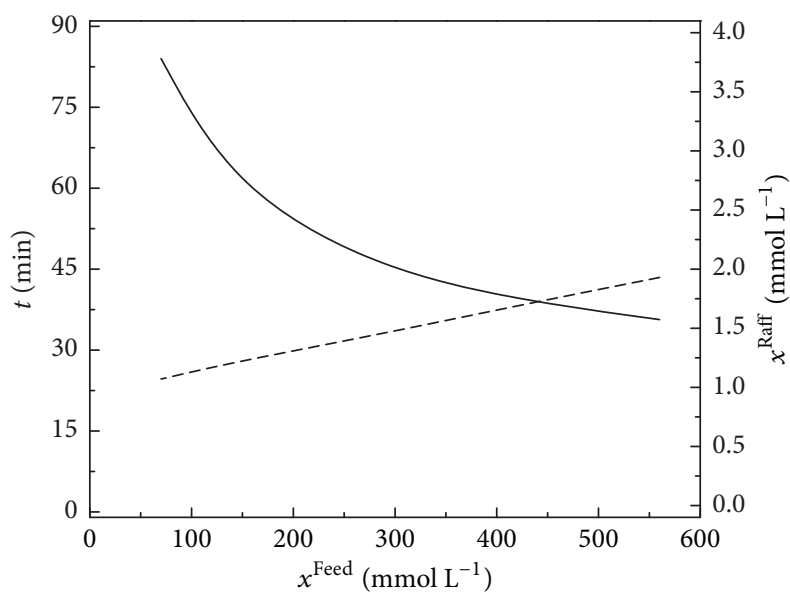

FIgURE 11: Adsorption unit cycle time (solid line) and raffinate concentration (dashed line) as a function of the initial concentration of the impurity in the oil phase $\left(x^{\mathrm{Feed}}\right)$, for a required residual concentration at the outlet of the adsorption column $\left(y^{\text {break }}\right)$ of $1.40 \mathrm{mmol} \mathrm{L}^{-1}$.

D6871, $0.6 \mathrm{mg} \mathrm{KOH} \mathrm{g}^{-1}$, that is, $10 \mathrm{mmol} \mathrm{L}^{-1}$, nine times the final value in the raffinate of the example. $0.6 \mathrm{mg} \mathrm{KOH} \mathrm{g}^{-1}$ is also the limit established for refined edible oil for human consumption (FAO CODEX-STAN 210, 1999) and therefore the extraction step is suitable for this application also, though it must be noted that ethanol would be more appropriate than methanol for a better compliance with health restrictions.

For simulating the adsorption column the set of equations (32)-(35) for the adsorption column was solved after analyzing the underlying hypotheses. The axial dispersion sometimes produces the broadening of the adsorption front due to the contribution of both molecular diffusion and dispersion caused by fluid flow [44]. The impact of the axial dispersion is assessed by the Peclet number $(\mathrm{Pe})$ small values indicating backmixing is important. According to Carberry [45], for Pe values much greater than 100, the flow can be considered plug flow type. Values of molecular diffusivity of oleic acid in methanol were calculated with Wilke-Chang equation. Axial diffusivity was calculated with the correlation of Wakao and Funazkri [46]. The Pe value was found to be equal to 3300 .

For the case of linear isotherm, a solution to ((32)-(35)) in the form $(q, y)=f(z, t)$ can be got by using the "quasilog normal distribution" (Q-LND) [47, 48]. In this case, it is assumed that the quasi-log normal probability density function can be used to represent the impulse response of the system. It has been demonstrated that the analytical solution and the Q-LND approximate solution are similar for a wide range of the model parameters and that deviations appear only for very low values of the residence time. This solution is used to plot the breakthrough curve of Figure 10.

In Figure 10, the concentration of the extract is $7.64 \mathrm{mmol} \mathrm{L}^{-1}$ and must be reduced to $0.7 \mathrm{mmol} \mathrm{L}^{-1}$. The concentration of impurity in the solvent at the column outlet is nonlinear function of time, and the initial concentration is about zero. An outlet concentration of $1.4 \mathrm{mmol} \mathrm{L}^{-1}$

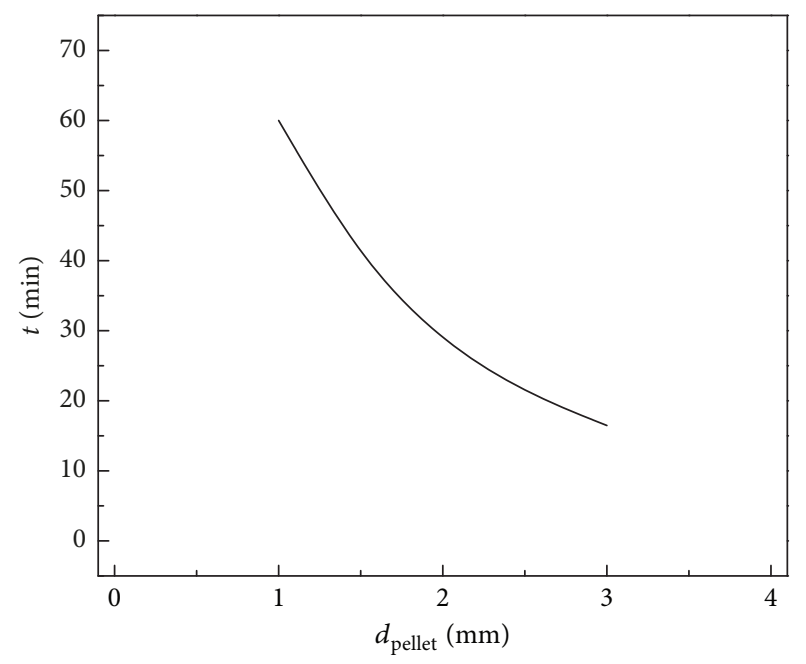

FIGURE 12: Adsorption unit cycle time as a function of the pellet diameter, for a required residual concentration at the outlet of the adsorption column ( $y^{\text {break }}$ ) of $1.40 \mathrm{mmol} \mathrm{L}^{-1}$ and impurity in the oil phase $\left(x^{\text {Feed }}\right)$ equal to $140 \mathrm{mmol} \mathrm{L}^{-1}$.

corresponds to an average concentration of solvent somewhat lower than $0.7 \mathrm{mmol} \mathrm{L}^{-1}$. $1.4 \mathrm{mmol} \mathrm{L}^{-1}$ could then be safely considered the column breakthrough condition $\left(y^{\text {break }}\right)$, the cycling time for the packed bed being of 1 hour. At this time, the average load of impurity on the adsorbent is $95 \mathrm{mmol} \mathrm{kg}$. The way of removing this load to regenerate the bed will be dealt later in detail.

$t^{\text {break }}$ will be a function of $x^{\text {feed }}$ and the desired purity of the solvent, for a given fixed operation condition of the extraction unit. This is illustrated in Figure 11. The adsorbent column must be maintained in operation until the breakthrough occurs. As expected $t^{\text {break }}$ decreases with higher concentration of impurity in the feed but the curve is enough soft to allow handling varying impurity concentration in the $100-600 \mathrm{mmol} \mathrm{L}^{-1}$ range with $t^{\text {break }}$ in the 30-60 min range. The concentration in the raffinate varies from 1 to $2 \mathrm{mmol} \mathrm{L}^{-1}$, which can be considered negligible.

The simulation runs of Figures 9, 10, and 11 were made with a pellet size of $1 \mathrm{~mm}$. For a bigger $12 \times 40$ meshes granular carbon, a maximum size of $1.5 \mathrm{~mm}$ can be found. For pelletized carbon, sizes of up to $3 \mathrm{~mm}$ are common. Particle size has a great influence on the cycling time, because the intrapellet diffusion mass transfer resistance $R_{D}$ is proportional to the square of the pellet radius. This is clear in the plot of Figure 12. The time of operation must be reduced from $1 \mathrm{~h}$ to about $15 \mathrm{~min}$ when the pellet size is increased from 1 to $3 \mathrm{~mm}$.

(2) Regeneration. An assessment of the regeneration of the solvent by evaporation/distillation and adsorption should be made. Distillation is the most common method but it requires a relatively high amount of energy. Adsorption was demonstrated to be a feasible regeneration method but it needs energy for desorbing the impurity from the adsorbent bed. A comparison of the amount of heat involved 
TABLE 4: Amounts of energy involved in the regeneration of the extract (in kJ per litre extract).

\begin{tabular}{lclc}
\hline \multicolumn{1}{c}{ Distillation } & & \multicolumn{1}{c}{ Adsorption } \\
\hline Step & Energy & Step & Energy \\
\hline $\begin{array}{l}\text { Heating to boiling point } \\
\text { Solvent evaporation }\end{array}$ & 63 & Adsorption (exothermal) & 0 \\
& 948 & Heating 1 bed volumes of methanol to $100^{\circ} \mathrm{C}$ & 22 \\
& & Desorption (endothermal) by elution of hot methanol & $<1$ \\
Total heat duty & 1011 & Heating to boiling point, evaporation of solvent of eluting stream & 174 \\
\hline
\end{tabular}

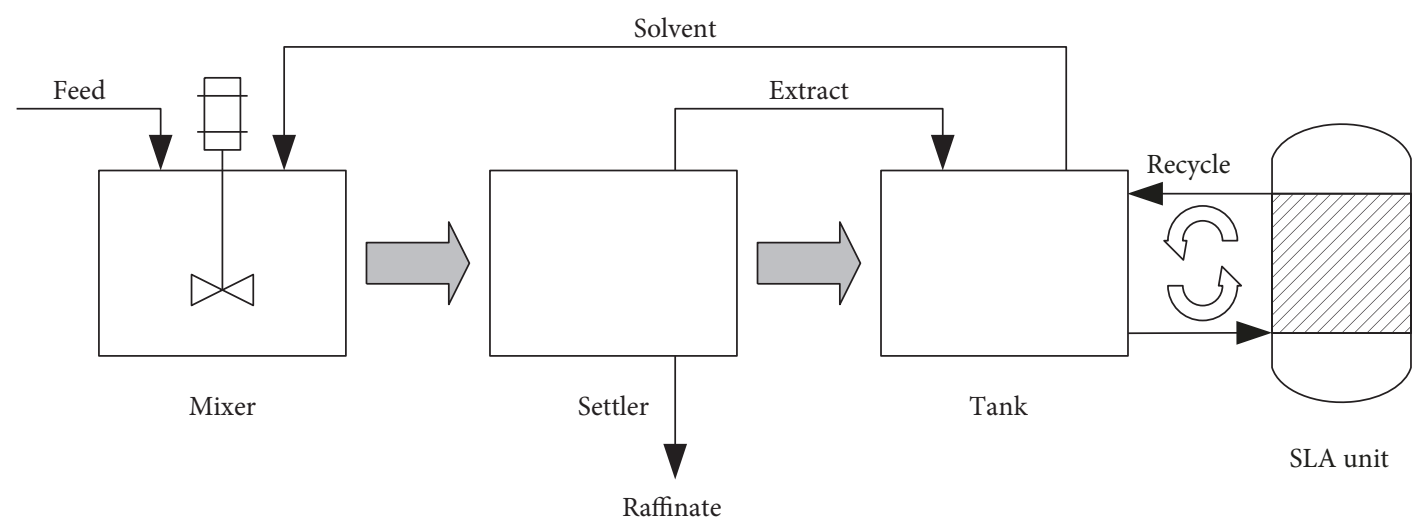

FIGURE 13: Flowsheet of a sequential discontinuous combination of extraction tank and adsorption column with fast recycle. Dimensions and process parameters: $W^{\text {Ads }}=1334 \mathrm{~kg}, V^{\text {Feed }}=0.24 \mathrm{~m}^{3}, V^{\text {Solv }}=4.8 \mathrm{~m}^{3}, x^{\text {Feed }}=140 \mathrm{mmol} \mathrm{L}^{-1}, T=303 \mathrm{~K}$.

in each case is done in Table 4 for the case of Figures 9 and 10. The information of these figures indicated that the regenerated solvent had an average concentration of oleic acid of $0.7 \mathrm{mmol} \mathrm{L}^{-1}$ and that the average concentration on the solid at breakthrough was about $95 \mathrm{mmol} \mathrm{kg}^{-1}$. The amount of solvent regenerated at $t^{\text {break }}$ was $4800 \mathrm{~L}$. If this volume had been regenerated by distillation the amount of heat would have been $1000 \mathrm{~kJ} \mathrm{~L}^{-1}$. This is considering that all the methanol is evaporated at $100 \%$ efficiency and that there are no schemes for heat recovery. In the case of the regeneration by adsorption, the heat is consumed in the heating of the eluting volume for the thermal swing, heating this same stream to the boiling point and evaporating the solvent in it to recover the free oleic acid. A temperature of regeneration of $100^{\circ} \mathrm{C}$ and an elution volume equal to 1 bed volume was chosen. These values permit achieving a residual concentration of impurity in the solid lower than 5\% of the original load before regeneration. With these parameters, the heat duty of the regeneration by adsorption amounts to about $151 \mathrm{~kJ} \mathrm{~L}^{-1}$, just a $15 \%$ of the classical regeneration. The regeneration temperature demands running the regeneration step with a little overpressure of 2.3 bar due to the high vapor pressure of methanol. In general terms, it was deduced that temperature of regeneration is the most influential variable, the elution volume having lower impact on the residual concentration of impurity in the solid. For simplicity of the involved calculation, regeneration will be assumed to be complete in what follows.
Time for regeneration was found experimentally. Residual oleic acid on the solid did not vary for time spans for regeneration higher than $2.5 \mathrm{~min}$.

Some other authors have used only flushing with solvent in order to regenerate the adsorbent. Yori et al. [49] removed glycerol from biodiesel by adsorption over a silica column and regenerated the bed by flushing with a small amount of methanol. In their case the great affinity of methanol for the adsorbed impurity (glycerol) was the crucial factor for regenerating the bed. In the studied case, a thermal swing is needed to help desorption.

(3) Simulation of a Batch Extraction/Adsorption Process. For this simulation, extraction tanks were chosen with a volume equal to that processed by the equipment of Figure 8 for $1 \mathrm{~h}$ operation (see Figure 13). This enables a comparison of performance and equipment requirements for similar throughput. In order to use completely discontinuous units, a column with fast recycle was programmed that obeys (30) and (31). Choosing a stirred tank with adsorbent suspended in the liquid would have yielded the same operation equations. However packed columns make regeneration easier. In order to have a short residence time and work as a perfectly mixed stirred tank, the recycling flow rate must be made fast enough. Reducing the residence time to a fraction of a minute makes this possible. For not making pressure drop an issue at this flow conditions the $\mathrm{L} / \mathrm{D}$ of the column should be low. 


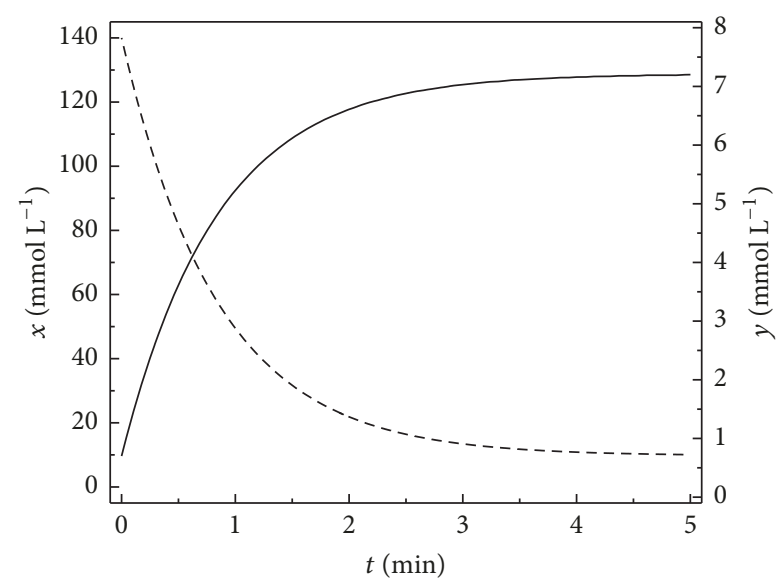

FIGURE 14: Plot of $x$ (dashed line) and $y$ (solid line) as a function of time in the batch extraction unit. $W^{\text {Ads }}=1334 \mathrm{~kg}, V^{\text {Feed }}=0.24 \mathrm{~m}^{3}$, $V^{\text {Solv }}=4.8 \mathrm{~m}^{3}, x^{\text {Feed }}=140 \mathrm{mmol} \mathrm{L}^{-1}, T=30^{\circ} \mathrm{C}$.

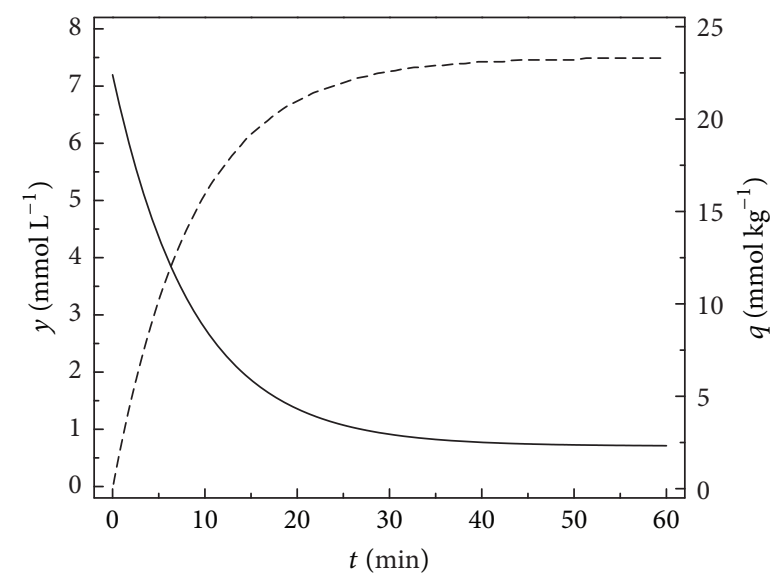

FIGURE 15: Plot of $y$ (solid line) and $q$ (dashed line) as a function of time in the adsorption column with recycle. $W^{\text {Ads }}=1334 \mathrm{~kg}, V^{\text {Feed }}=$ $240 \mathrm{~L}, V^{\text {Solv }}=1800 \mathrm{~L}, y^{\text {Raff }}=7.2 \mathrm{mmol} \mathrm{L}^{-1}, T=30^{\circ} \mathrm{C}$.

Results of the simulation are given in Figure 14. The concentration of the impurity can be reduced from 140 to about $10 \mathrm{mmol} \mathrm{L}^{-1}$. This is worse than the final value of the countercurrent column, $1.1 \mathrm{mmol} \mathrm{L}^{-1}$, and is a consequence of the unfavorable behavior of perfectly mixed systems with equilibrium restrictions. Two stages would be necessary for achieving the final raffinate concentration of Figure 9.

The results of Figure 15 bring similar conclusions as in the case of the batch extraction unit. The performance of the column with fast recycle is worse than that seen for the column with once-through flow. Although the extract to be refined has a similar concentration of impurity $\left(7.2 \mathrm{mmol} \mathrm{L}^{-1}\right)$, an outlet solvent concentration of $0.7 \mathrm{mmol}^{-1}$ like in Figure 10 can only be achieved by increasing the mass of adsorbent 3.6 times. This is also explained by the unfavorable behavior of perfectly mixed systems with equilibrium restrictions.

The comparison of the saturation time values for both the extraction and adsorption column shows that the extraction tank has a saturation time of about $5 \mathrm{~min}$ and the packed

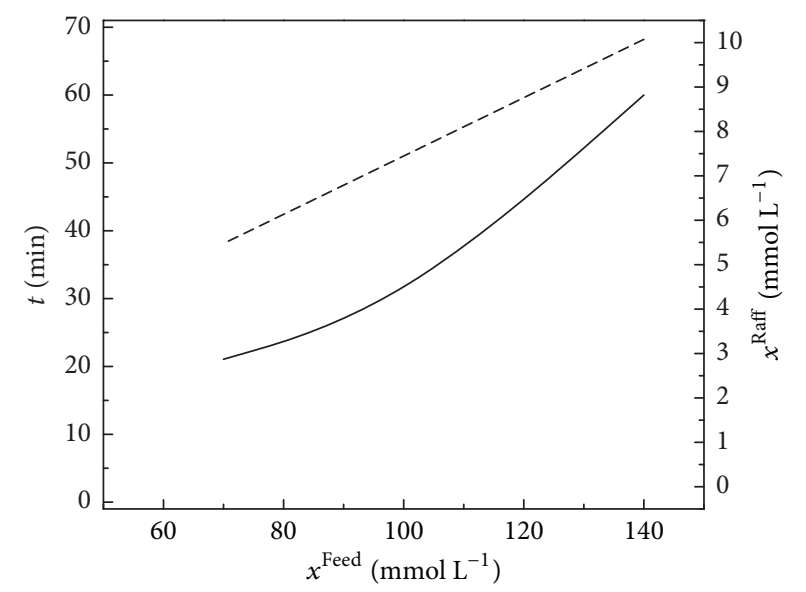

FIGURE 16: Adsorption time (solid line) as a function of the initial concentration of the impurity in the oil phase $\left(x^{\mathrm{Feed}}\right)$, for a required residual concentration at the outlet of the adsorption column $\left(y^{\text {break }}\right)$ of $0.70 \mathrm{mmol} \mathrm{L}^{-1}$. Resulting $x^{\text {Raff }}$ as a function of $x^{\text {Feed }}$ (dashed line). $W^{\text {Ads }}=1334 \mathrm{~kg}$.

bed about $40 \mathrm{~min}$. This is the result obtained for a volume of $4800 \mathrm{~L}$ liquid phase in the extractor and a volume of about $2600 \mathrm{~L}$ of packed bed. It can be deduced that the throughput of each unit per unit volume is different, about $200 \mathrm{~L} \mathrm{~min}^{-1}$ per $\mathrm{L}$ of process vessel for the extractor and 0.05 for the adsorber. These values are not too different as it could be expected from the slow kinetics of adsorption and could be the results of a compensation with a high available surface area for the chosen adsorbent.

A comparison of all characteristic times must also include the settling time of the decanter. At $50^{\circ} \mathrm{C}$ this is about $1 \mathrm{~h}$, more similar to the saturation time of the adsorber. However, the packed bed should be operated at a conveniently low temperature, like $30^{\circ} \mathrm{C}$, to have a favorable adsorbing capacity. At $30^{\circ} \mathrm{C}$ the settling time increases to about $3 \mathrm{~h}$, making this step the slowest of the process.

A plot of the necessary minimum adsorption time as a function of the feed impurity concentration is included in Figure 16. The same trends of Figure 11 are seen. The concentration of the raffinate increases when the concentration of impurity in the feed is increased. Keeping the raffinate at a constant composition can only be achieved by increasing the solvent-to-feed ratio in the extraction unit. The operation time for the adsorption column increases when the concentration of impurity in the feed increases. This is due to the higher concentration of impurity that demands more time to be removed.

\section{Conclusions}

The deacidification of sunflower oil by extraction with methanol and regeneration of the solvent by adsorption on activated carbon were tried. The method is considered useful for the food and biodiesel industries as a means of economically achieving low impurity levels in raffinate streams in systems with a high solvent-to-feed ratio. 
Equations for the design of extractors and packed columns, both in continuous and discontinuous mode, were developed. Suitable equations for design were written from general principles, highlighting similarities in formulation for driving forces, mass transfer rates, thermodynamic and kinetic parameters. A comparison of ranges of thermodynamic and kinetic parameters seems to indicate that the matching of extraction and adsorption units needs to accommodate the fast kinetics of extraction with the relatively slow kinetics of adsorption, and the relatively high affinity of adsorbents per unit mass with the low capacity of most solvents per unit volume. In this sense a solution is the cyclic operation of a column adsorber of adequate size.

Deacidification by extraction with methanol can be carried out with relatively high efficiency due to a fairly high value of the partition coefficient for oleic acid between the polar and organic phase, with values of $m=0.74$ a 0.93 in the range of temperatures 30 a $50^{\circ} \mathrm{C}$. Extraction in a countercurrent column with a solvent-to-feed ratio of 20 allows reduction of the acidity of an oil with $4.4 \%$ acidity to a final value of $0.04 \%$, adequate for its use as a feedstock for the production of biodiesel with the alkali-catalyzed process, its use as edible oil for human consumption, or its use as dielectric biodegradable oil. Extraction permits a maximum yield of oil and the recovery of the fatty acid impurity.

Typical operation of a stirred tank extraction unit yielded a value of the global coefficient for mass transfer $a K_{L}$ of $0.75 \mathrm{~min}^{-1}$ (methanol side). Fast kinetics of extraction permitted the operation of a stirred tank extraction unit with a saturation time of about $5 \mathrm{~min}$. This was faster than the characteristic time for saturation of a batch adsorption column that had a value of about $1 \mathrm{~h}$. However, a totally discontinuous process needs also of a decanter that had big settling times, making it the slowest step of the process.

The extraction/adsorption combination seems well suited for extraction operations with a high solvent-to-feed ratio in which solvent regeneration by distillation becomes prohibitive due to a lower vapor pressure of the impurity to be removed/recovered, thus demanding evaporation of large amounts of solvent. It was demonstrated, with the example of sunflower oil deacidification, that, for a solvent-to-feed ratio of 20 , the heat duty of a distillation-based solvent regeneration could be as large as $1011 \mathrm{~kJ}$ per litre of solvent. A regeneration process based on adsorption needs of a heat duty much lower, of about $174 \mathrm{~kJ}$ per litre solvent, the heat duty mainly being related to the thermal swing of the packed bed.

Applications for extraction with a high solvent-to-feed ratio for which an extraction/adsorption combination would be convenient could be those using a solvent with high selectivity but low affinity for the impurity, or "polishing" operations with a low driving force due to the high dilution of the impurity in the feed.

The extraction/adsorption system is amenable for both continuous and discontinuous operation. However, the continuous operation has a higher efficiency due to the intrinsic advantages of plug flow as compared to perfect mixing, for systems in thermodynamic equilibrium is a limitation.
For adsorption the main mass transfer resistance is intrapellet diffusion. In this sense small adsorbent particles improve the turnover of the process and increase the percentage of utilization of the adsorbent volume.

\section{Conflicts of Interest}

The authors declare that there are no conflicts of interest regarding the publication of this paper.

\section{Acknowledgments}

This work was performed with the funding of CONICET (PIP Grants 11220130100457CO and 11420110100235CO), Universidad Nacional del Litoral (CAI + D Grant 50420150100074LI), and Universidad Nacional de Jujuy (SeCTER 08/D138).

\section{References}

[1] M. S. Cuevas, C. E. C. Rodrigues, and A. J. A. Meirelles, "Effect of solvent hydration and temperature in the deacidification process of sunflower oil using ethanol," Journal of Food Engineering, vol. 95, no. 2, pp. 291-297, 2009.

[2] C. E. C. Rodrigues, C. B. Gonçalves, E. Batista, and A. J. A. Meirelles, "Deacidification of Vegetable Oils by Solvent Extraction," Recent Patents on Engineering, vol. 1, no. 1, pp. 95-102, 2007.

[3] S. Turkay and H. Civelekoglu, "Deacidification of sulfur olive oil. I. Single-stage liquid-liquid extraction of miscella with ethyl alcohol," Journal of the American Oil Chemists' Society, vol. 68, no. 2, pp. 83-86, 1991.

[4] R. A. Carr, "Degumming and refining practices in the U.S.", Journal of the American Oil Chemists' Society, vol. 53, no. 6, pp. 347-352, 1976.

[5] V. Kale, S. P. R. Katikaneni, and M. Cheryan, "Deacidifying rice bran oil by solvent extraction and membrane technology," Journal of the American Oil Chemists' Society, vol. 76, no. 6, pp. 723-727, 1999.

[6] S. S. Koseoglu, "Membrane technology for edible oil refining," Oils \& Fats International, vol. 5, pp. 16-21, 1991.

[7] N. Othman, Z. A. Manan, S. R. Wan Alwi, and M. R. Sarmidi, "A review of extraction technology for carotenoids and vitamin e recovery from palm oil," Journal of Applied Sciences, vol. 10, no. 12, pp. 1187-1191, 2010.

[8] C. Vera, M. Busto, J. Yori et al., "Adsorption in Biodiesel Refining - A Review," in Biodiesel - Feedstocks and Processing Technologies, Intech Open Access Publishers, 2011.

[9] H. Zhang, A. Li, J. Sun, and P. Li, "Adsorption of amphoteric aromatic compounds by hyper-cross-linked resins with amino groups and sulfonic groups," Chemical Engineering Journal, vol. 217, pp. 354-362, 2013.

[10] M. Yuan, S. Tong, S. Zhao, and C. Q. Jia, "Adsorption of polycyclic aromatic hydrocarbons from water using petroleum coke-derived porous carbon," Journal of Hazardous Materials, vol. 181, no. 1-3, pp. 1115-1120, 2010.

[11] M. Muzic, K. Sertic-Bionda, Z. Gomzi, S. Podolski, and S. Telen, "Study of diesel fuel desulfurization by adsorption," Chemical Engineering Research and Design, vol. 88, no. 4, pp. 487-495, 2010. 
[12] M. L. M. Oliveira, A. A. L. Miranda, C. M. B. M. Barbosa, C. L. Cavalcante Jr., D. C. S. Azevedo, and E. Rodriguez-Castellon, "Adsorption of thiophene and toluene on $\mathrm{NaY}$ zeolites exchanged with $\mathrm{Ag}(\mathrm{I}), \mathrm{Ni}(\mathrm{II})$ and $\mathrm{Zn}(\mathrm{II})$, , Fuel, vol. 88, no. 10, pp. 1885-1892, 2009.

[13] L. Zou, B. Han, H. Yan, K. L. Kasperski, Y. Xu, and L. G. Hepler, "Enthalpy of adsorption and isotherms for adsorption of naphthenic acid onto clays," Journal of Colloid and Interface Science, vol. 190, no. 2, pp. 472-475, 1997.

[14] F. Wang, J. J.-H. Haftka, T. L. Sinnige, J. L. M. Hermens, and W. Chen, "Adsorption of polar, nonpolar, and substituted aromatics to colloidal graphene oxide nanoparticles," Environmental Pollution, vol. 186, pp. 226-233, 2014.

[15] J. Saien and S. Daliri, "Mass transfer coefficient in liquid-liquid extraction and the influence of aqueous phase $\mathrm{pH}$," Industrial \& Engineering Chemistry Research, vol. 47, no. 1, pp. 171-175, 2008.

[16] E. Batista, S. Monnerat, K. Kato, L. Stragevitch, and A. J. A. Meirelles, "Liquid-liquid equilibrium for systems of canola oil, oleic acid, and short-chain alcohols," Journal of Chemical \& Engineering Data, vol. 44, no. 6, pp. 1360-1364, 1999.

[17] C. L. Munson and C. J. Klng, "Factors Influencing Solvent Selection for Extraction of Ethanol from Aqueous Solutions," Industrial \& Engineering Chemistry Process Design and Development, vol. 23, no. 1, pp. 109-115, 1984.

[18] H. D. Schindler and R. E. Treybal, "Continuous-phase masstransfer coefficients for liquid extraction in agitated vessels," AIChE Journal, vol. 14, no. 5, pp. 790-798, 1968.

[19] B. Schuur, W. J. Jansma, J. G. M. Winkelman, and H. J. Heeres, "Determination of the interfacial area of a continuous integrated mixer/separator (CINC) using a chemical reaction method," Chemical Engineering and Processing: Process Intensification, vol. 47, no. 9-10, pp. 1484-1491, 2008.

[20] R. P. Verma and M. M. Sharma, "Mass transfer in packed liquidliquid extraction columns," Chemical Engineering Science, vol. 30, no. 3, pp. 279-292, 1975.

[21] B. Xu, W. Cai, X. Liu, and X. Zhang, "Mass transfer behavior of liquid-liquid slug flow in circular cross-section microchannel," Chemical Engineering Research and Design, vol. 91, no. 7, pp. 1203-1211, 2013.

[22] S.-S. Ashrafmansouri and M. Nasr Esfahany, "The influence of silica nanoparticles on hydrodynamics and mass transfer in spray liquid-liquid extraction column," Separation and Purification Technology, vol. 151, Article ID 12450, pp. 74-81, 2015.

[23] H. Bahmanyar and M. J. Slater, "Studies of drop break-up in liquid-liquid systems in a rotating disc contactor. Part I: Conditions of no mass transfer," Chemical Engineering \& Technology, vol. 14, no. 2, pp. 79-89, 1991.

[24] Z. Azizi, A. Rahbar, and H. Bahmanyar, "Investigation of packing effect on mass transfer coefficient in a single drop liquid extraction column," Iranian Journal of Chemical Engineering, vol. 7, no. 4, pp. 3-11, 2010.

[25] E. Glueckauf and J. I. Coates, "Theory of chromatography. Part IV. The influence of incomplete equilibrium on the front boundary of chromatograms and on the effectiveness of separation," Journal of the Chemical Society (Resumed), pp. 1315-1321, 1947.

[26] M. Otero, C. A. Grande, and A. E. Rodrigues, "Adsorption of salicylic acid onto polymeric adsorbents and activated charcoal," Reactive and Functional Polymers, vol. 60, no. 1-3, pp. 203-213, 2004.

[27] D. M. Ruthven and S. Farooq, "Air separation by pressure swing adsorption," Gas Separation and Purification, vol. 4, no. 3, pp. 141-148, 1990.
[28] C. I. Koncsag and A. Barbulescu, "Liquid-Liquid Extraction with and without a Chemical Reaction," in Mass Transfer in Multiphase Systems and its Applications, Chapter 10, InTech Open Access Publishers, 2011.

[29] T. Sankarshana, S. Ilaiah, and U. Virendra, Liquid-Liquid Extraction: Comparison in Micro and Macro Systems, AIChE Annual Meeting, 2013.

[30] F. Nosratinia, M. R. Omidkhah, D. Bastani, and A. A. Saifkordi, "Investigation of mass transfer coefficient under jetting conditions in a liquid-liquid extraction system," Iranian Journal of Chemistry and Chemical Engineering, vol. 29, no. 1, pp. 1-12, 2010.

[31] C. J. Geankoplis and A. N. Hixson, "Mass Transfer Coefficients in an Extraction Spray Tower," Industrial \& Engineering Chemistry, vol. 42, no. 6, pp. 1141-1151, 1950.

[32] A. Salimi-Khorshidi, H. Abolghasemi, A. Khakpay, Z. Kheirjooy, and M. Esmaieli, "Spray and packed liquid-liquid extraction columns: Drop size and dispersed phase mass transfer," Asia-Pacific Journal of Chemical Engineering, vol. 8, no. 6, pp. 940-949, 2013.

[33] G. A. Hughmark, "Liquid-liquid spray column drop size, holdup, and continuous phase mass transfer," Industrial \& Engineering Chemistry Fundamentals, vol. 6, no. 3, pp. 408-413, 1967.

[34] C. L. Ruby and J. C. Elgin, "Mass transfer between liquid drops and a continuous phase in a countercurrent fluidized system: liquidliquid extraction in a spray tower," Chemical Engineering Progress Symposium, vol. 51, pp. 17-29, 1955.

[35] S. Kang and B. Xing, "Adsorption of dicarboxylic acids by clay minerals as examined by in situ ATR-FTIR and ex situ DRIFT," Langmuir, vol. 23, no. 13, pp. 7024-7031, 2007.

[36] C. D. Hatch, R. V. Gough, and M. A. Tblbert, "Heterogeneous uptake of the $\mathrm{C} 1$ to $\mathrm{C} 4$ organic acids on a swelling clay mineral," Atmospheric Chemistry and Physics, vol. 7, no. 16, pp. 44454458, 2007.

[37] N. Kanazawa, K. Urano, N. Kokado, and Y. Urushigawa, "Exchange characteristics of monocarboxylic acids and monosulfonic acids onto anion-exchange resins," Journal of Colloid and Interface Science, vol. 271, no. 1, pp. 20-27, 2004.

[38] E. Suescún-Mathieu, A. Bautista-Carrizosa, R. Sierra, L. Giraldo, and J. C. Moreno-Piraján, "Carboxylic acid recovery from aqueous solutions by activated carbon produced from sugarcane bagasse," Adsorption, vol. 20, no. 8, pp. 935-943, 2014.

[39] Y. S. Aşçı and I. M. Hasdemir, "Removal of Some Carboxylic Acids from Aqueous Solutions by Hydrogels," Journal Chemical \& Engineerind data, vol. 53, pp. 2351-2355, 2008.

[40] A. Li, Q. Zhang, G. Zhang, J. Chen, Z. Fei, and F. Liu, "Adsorption of phenolic compounds from aqueous solutions by a water-compatible hypercrosslinked polymeric adsorbent," Chemosphere, vol. 47, no. 9, pp. 981-989, 2002.

[41] M. S. Chiou and H. Y. Li, "Adsorption behavior of reactive dye in aqueous solution on chemical cross-linked chitosan beads," Chemosphere, vol. 50, no. 8, pp. 1095-1105, 2003.

[42] O. Ilgen and H. S. Dulger, "Removal of oleic acid from sunflower oil on zeolite 13X: Kinetics, equilibrium and thermodynamic studies," Industrial Crops and Products, vol. 81, pp. 66-71, 2016.

[43] R. Gayler, N. W. Roberts, and H. R. Pratt, "liquid-liquid extraction: Part IV. A further study of hold-up in packed columns," Chemical Engineering Research and Design, vol. 31, pp. 57-68, 1953.

[44] D. M. Ruthven, Principles of Adsorption and Adsorption Processes, John Wiley \& Sons Inc, New York, NY, USA, 1984. 
[45] J. J. Carberry, Chemical and Catalytic Reaction Engineering, McGraw Hill, New York, NY, USA, 1976.

[46] N. Wakao and T. Funazkri, "Effect of fluid dispersion coefficients on particle-to-fluid mass transfer coefficients in packed beds. Correlation of sherwood numbers," Chemical Engineering Science, vol. 33, no. 10, pp. 1375-1384, 1978.

[47] M. E. Sigrist, H. R. Beldomenico, E. E. Tarifa, C. L. Pieck, and C. R. Vera, "Modelling diffusion and adsorption of As species in Fe/GAC adsorbent beds," Journal of Chemical Technology and Biotechnology, vol. 86, no. 10, pp. 1256-1264, 2011.

[48] G.-H. Xiu, T. Nitta, P. Li, and G. Jin, "Breakthrough Curves for Fixed-Bed Adsorbers: Quasi-Lognormal Distribution Approximation," AIChE Journal, vol. 43, no. 4, pp. 979-985, 1997.

[49] J. C. Yori, S. A. D’Ippolito, C. L. Pieck, and C. R. Vera, "Deglycerolization of biodiesel streams by adsorption over silica beds," Energy \& Fuels, vol. 21, no. 1, pp. 347-353, 2007. 


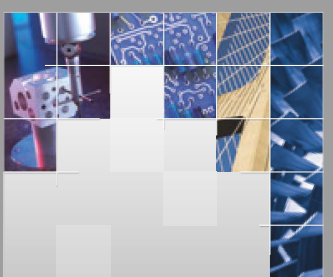

\section{Enfincering}
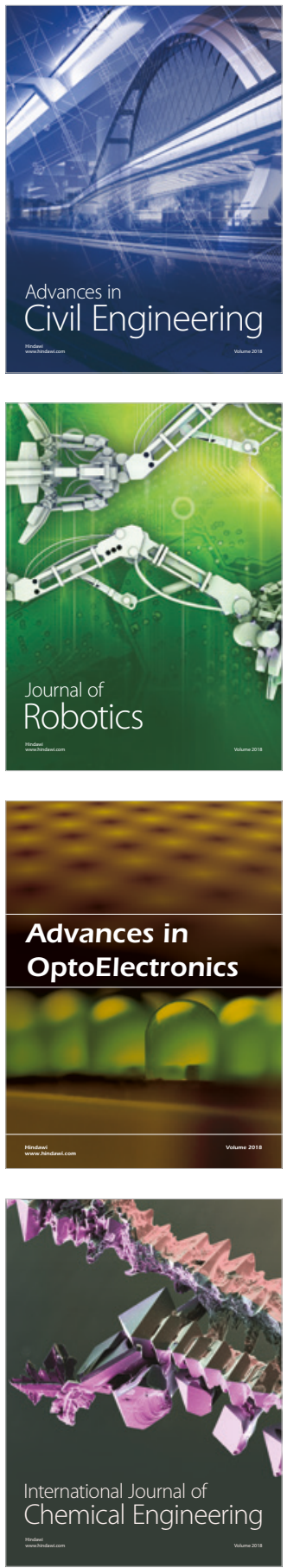

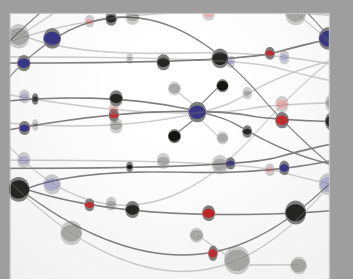

\section{Rotating \\ Machinery}

The Scientific World Journal

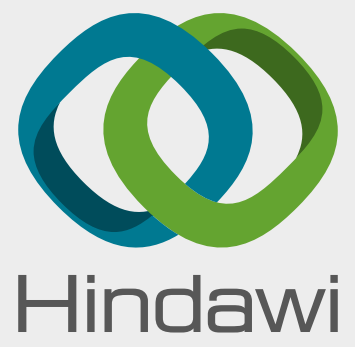

Submit your manuscripts at

www.hindawi.com
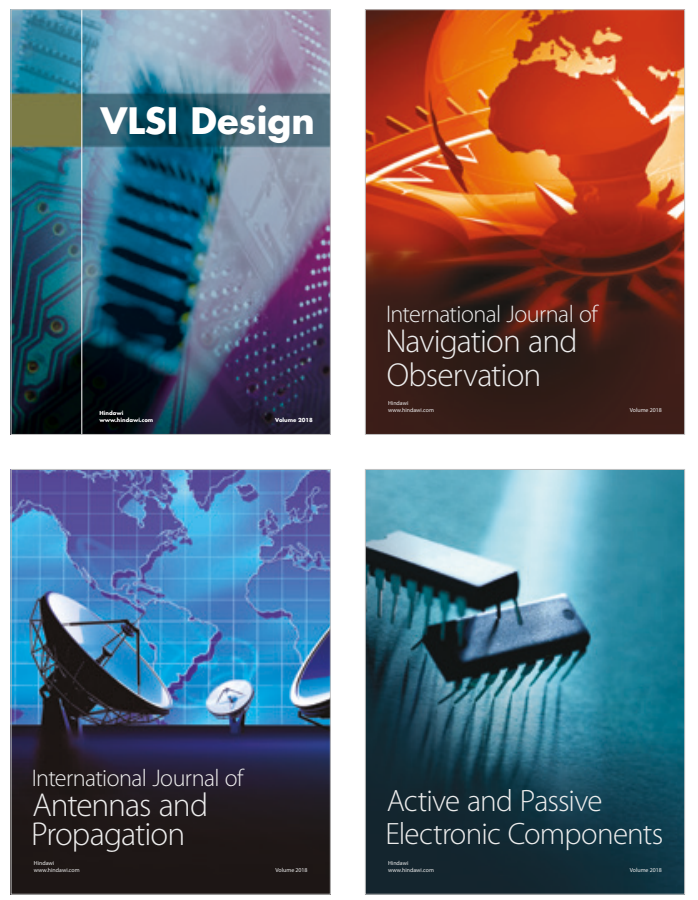
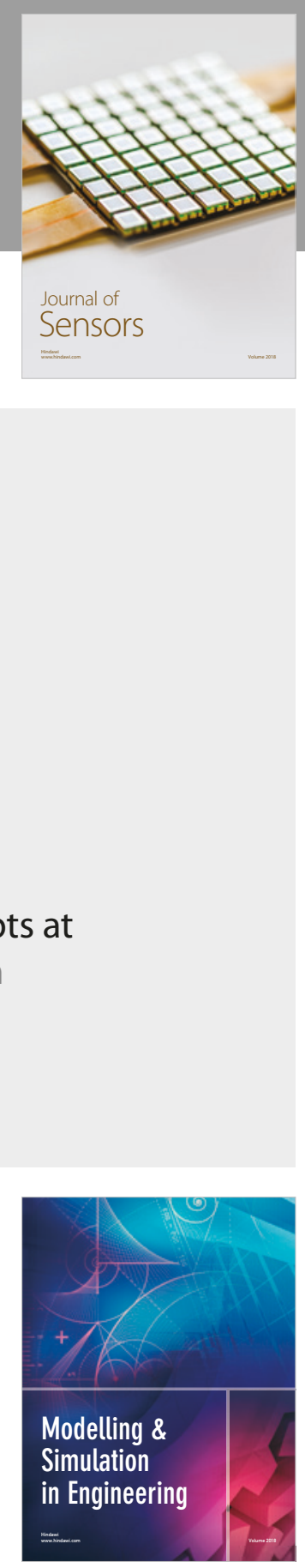

\section{Advances \\ Multimedia}
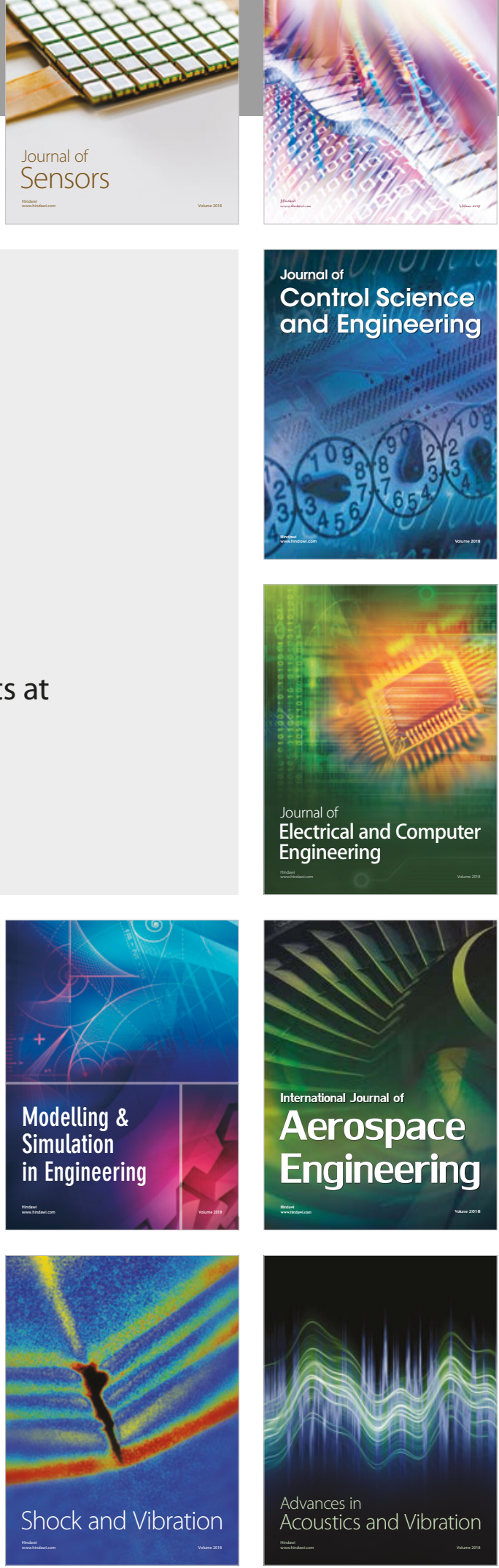